\title{
SMOOTH AND PEAKED SOLITONS OF THE CAMASSA-HOLM EQUATION AND APPLICATIONS
}

\author{
DARRYL HOLM AND ROSSEN IVANOV
}

Communicated by Alexandar B. Yanovski

\begin{abstract}
The relations between smooth and peaked soliton solutions are reviewed for the Camassa-Holm $(\mathrm{CH})$ shallow water wave equation in one spatial dimension. The canonical Hamiltonian formulation of the $\mathrm{CH}$ equation in action-angle variables is expressed for solitons by using the scattering data for its associated isospectral eigenvalue problem, rephrased as a Riemann-Hilbert problem. The momentum map from the action-angle scattering variables $T^{*}\left(\mathbb{T}^{N}\right)$ to the flow momentum provides the Eulerian representation of the $N$-soliton solution of $\mathrm{CH}$ in terms of the scattering data and squared eigenfunctions of its isospectral eigenvalue problem. The dispersionless limit of the $\mathrm{CH}$ equation and its resulting peakon solutions are examined by using an asymptotic expansion in the dispersion parameter. The peakon solutions of the dispersionless $\mathrm{CH}$ equation in one dimension are shown to generalize in higher dimensions to peakon wave-front solutions of the EPDiff equation whose associated momentum is supported on smoothly embedded subspaces. The Eulerian representations of the singular solutions of both $\mathrm{CH}$ and EPDiff are given by the (cotangent-lift) momentum maps arising from the left action of the diffeomorphisms on smoothly embedded subspaces.
\end{abstract}

\section{Contents}

1 Shallow Water Background for the CH Equation 14

2 Soliton Solutions of CH Equation from Inverse Scattering 18

2.1 Inverse Scattering for the KdV Equation . . . . . . . . . . . 18

2.2 Inverse Scattering for $\mathrm{CH}$ Solitons with Dispersion . . . . . . . . 19

2.3 Parametric Form of the Dispersive CH Soliton Solution . . . . . . 23

2.4 Relation to KdV Hierarchy . . . . . . . . . . . . . . 24

3 Momentum Map Formulation with Action-angle Variables 25

4 Peakons 27 
4.1 Peakons: the Singular Solution Ansatz . . . . . . . . . . . . . 27

4.2 Integrable Peakon Dynamics of $\mathrm{CH} \ldots \ldots . \ldots 28$

5 Peakon Limit of the CH Soliton Solutions 30

6 Superintegrability of the Peakon System 34

7 Other Singular Solutions: the Dispersionless $b$-equation 35

8 Euler-Poincaré Theory in Higher Dimensions 37

9 Two Open Problems 43

\section{Shallow Water Background for the CH Equation}

Euler's equations for irrotational incompressible ideal fluid motion under gravity with a free surface have an asymptotic expansion for shallow water waves that contains two small parameters, $\epsilon$ and $\delta^{2}$, with ordering $\epsilon \geq \delta^{2}$. These small parameters are $\epsilon=a / h_{0}$ (the ratio of wave amplitude to mean depth) and $\delta^{2}=\left(h_{0} / l_{x}\right)^{2}$ (the squared ratio of mean depth to horizontal length, or wavelength). Euler's equations are made non-dimensional by introducing $x=l_{x} x^{\prime}$ for horizontal position, $z=h_{0} z^{\prime}$ for vertical position, $t=\left(l_{x} / c_{0}\right) t^{\prime}$ for time, $\eta=a \eta^{\prime}$ for surface elevation and $\varphi=\left(g l_{x} a / c_{0}\right) \varphi^{\prime}$ for velocity potential, where $c_{0}=\sqrt{g h_{0}}$ is the mean wave speed and $g$ is the constant gravity. The quantity $\sigma=\sigma^{\prime} /\left(h_{0} \rho c_{0}^{2}\right)$ is the dimensionless Bond number, in which $\rho$ is the mass density of the fluid and $\sigma^{\prime}$ is its surface tension, both of which are taken to be constants. After dropping primes, this asymptotic expansion yields the nondimensional Korteweg-de Vries $(\mathrm{KdV})$ equation for the horizontal velocity variable $u=\varphi_{x}(x, t)$ at linear order in the small dimensionless ratios $\epsilon$ and $\delta^{2}$, as the left hand side of

$$
u_{t}+u_{x}+\frac{3 \epsilon}{2} u u_{x}+\frac{\delta^{2}}{6}(1-3 \sigma) u_{x x x}=O\left(\epsilon \delta^{2}\right) .
$$

Here, partial derivatives are denoted using subscripts, and boundary conditions are $u=0$ and $u_{x}=0$ at spatial infinity on the real line. The famous traveling wave solutions $\operatorname{sech}^{2}(x-c t)$ (the solitons) for KdV (1) arise in a balance between its (weakly) nonlinear steepening and its third-order linear dispersion, when the quadratic terms in $\epsilon$ and $\delta^{2}$ on its right hand side are neglected.

On the right hand side of equation (1), a normal form transformation due to Kodama [41] has been used to remove the other possible quadratic terms of order 
$O\left(\epsilon^{2}\right)$ and $O\left(\delta^{4}\right)$. The remaining quadratic correction terms in the $\mathrm{KdV}$ equation (1) may be collected at order $O\left(\epsilon \delta^{2}\right)$. These terms may be expressed, after introducing a "momentum variable"

$$
m=u-\nu \delta^{2} u_{x x}
$$

and neglecting terms of cubic order in $\epsilon$ and $\delta^{2}$, as

$$
m_{t}+m_{x}+\frac{\epsilon}{2}\left(u m_{x}+b m u_{x}\right)+\frac{\delta^{2}}{6}(1-3 \sigma) u_{x x x}=0 .
$$

In the momentum variable (2), the parameter $\nu$ is given in [21] by

$$
\nu=\frac{19-30 \sigma-45 \sigma^{2}}{60(1-3 \sigma)} .
$$

Thus, the effects of $\delta^{2}$-dispersion also enter the nonlinear terms. After restoring dimensions in equation (3) and rescaling velocity $u$ by $(b+1)$, the following " $b$ equation" emerges

$$
m_{t}+c_{0} m_{x}+u m_{x}+b m u_{x}+\Gamma u_{x x x}=0
$$

where $m=u-\alpha^{2} u_{x x}$ is the dimensional momentum variable, and the constants $\alpha^{2}$ and $\Gamma / c_{0}$ are squares of length scales. When $\alpha^{2} \rightarrow 0$, one recovers $\mathrm{KdV}$ from the $b$-equation (4), up to a rescaling of velocity. Any value of the parameter $b \neq-1$ may be achieved in equation (4) by an appropriate Kodama transformation [21].

As we have emphasized, the values of the coefficients in the asymptotic analysis of shallow water waves at quadratic order in their two small parameters only hold, modulo the Kodama normal-form transformations. Hence, these transformations may be used to advance the analysis and thereby gain insight, by optimizing the choices of these coefficients. The freedom introduced by the Kodama transformations among asymptotically equivalent equations at quadratic order in $\epsilon$ and $\delta^{2}$ also helps to answer the perennial question, "why are integrable equations so ubiquitous when one uses asymptotics in modeling?" Namely, there may be special values of the free parameters in the normal-form transformations of the asymptotics that allow integrability.

\section{Integrable Cases of the $b$-equation}

The cases $b=2$ and $b=3$ are special values. For these values, the $b$-equation becomes completely integrable as a Hamiltonian system. For $b=2$, equation (4) specializes to the integrable $\mathrm{CH}$ equation of Camassa and Holm [9]. The case 
$b=3$ in (4) recovers the integrable DP equation of Degasperis and Procesi [19]. These two cases exhaust the integrable candidates for (4), as was shown using Painlevé analysis. The $b$-family of equations (4) was also shown in [47] to admit the symmetry conditions necessary for integrability, only in the cases $b=2$ for $\mathrm{CH}$ and $b=3$ for DP.

The $b$-equation (4) with $b=2$ was first derived in Camassa and Holm [9] by using asymptotic expansions directly in the Hamiltonian for Euler's equations governing inviscid incompressible flow in the shallow water regime. In this analysis, the $\mathrm{CH}$ equation was shown to be bi-Hamiltonian and thereby was found to be completely integrable by the inverse scattering transform (IST) on the real line. This development of IST for CH equation (4) with $b=2$ is discussed in Section 2.

Camassa and Holm [9] also discovered the remarkable peaked soliton (peakon) solutions of $(5,35)$ for the $\mathrm{CH}$ equation on the real line, given by $(4)$ in the case $b=2$. The peakons arise as solutions of (4), when $c_{0}=0$ and $\Gamma=0$ in the absence of linear dispersion. Peakons move at a speed equal to their maximum height, at which they have a sharp peak (jump in derivative). The single peakon solution is

$$
u(x, t)=c \mathrm{e}^{-|x-c t| / \alpha} .
$$

Unlike the KdV soliton, the peakon speed is independent of its width $(\alpha)$. Periodic peakon solutions of $\mathrm{CH}$ were treated in Alber et al [3]. There, the sharp peaks of periodic peakons were associated with billiards reflecting at the boundary of an elliptical domain. These billiard solutions for the periodic peakons arise from geodesic motion on a tri-axial ellipsoid, in the limit that one of its axes shrinks to zero length.

The $\mathrm{CH}$ equation was found after its derivation as a shallow water equation in [9] to fit into a class of integrable equations derived previously by using hereditary symmetries in Fokas and Fuchssteiner [23]. See Fuchssteiner [25] for an insightful history of how the shallow water equation (4) in the integrable case with $b=2$ relates to the mathematical theory of hereditary symmetries.

Equation (4) with $b=2$ was recently re-derived as a shallow water equation by using asymptotic methods in three different approaches in Dullin et al [21], in Fokas and Liu [24] and also in Johnson [38]. These three derivations all used different variants of the method of asymptotic expansions for shallow water waves in the absence of surface tension. Only the derivation in Dullin et al [21] took advantage of the parametric freedom in the Kodama normal-form transformations of the asymptotic expansion results at quadratic order.

The effects of the parameter $b$ on the solutions of equation (4) were investigated in Holm and Staley [36], where $b$ was treated as a bifurcation parameter, in the limiting case when the linear dispersion coefficients are set to $c_{0}=0$ and $\Gamma=0$. 
This limiting case allows a variety of special solutions for different ranges of the values of $b$, in which the two nonlinear terms in equation (4) balance each other in the absence of linear dispersion.

Since its first appearance in [9], the $\mathrm{CH}$ equation has been the centre of a confluence of scientific endeavors that includes water waves, integrable systems, partial differential equation (PDE) analysis, asymptotics, geometry and Lie groups. This confluence has led to a continued interest and opportunities for contributions from many different fields in mathematics. The interest in $\mathrm{CH}$ solutions may be measured by noticing that the entry 'peakon' has acquired many thousands of Google hits.

\section{Plan of the Paper}

This paper aims to review some of the geometric highlights of recent work on the $\mathrm{CH}$ equation. It is certainly not exhaustive. It mainly focuses on comparing the soliton theory for smooth $\mathrm{CH}$ solutions with the peakon theory for its singular solutions in the dispersionless limit. Section 2 briefly explains the application of the method of the IST for obtaining the soliton solutions of $\mathrm{CH}$ equation. The set of scattering data is introduced and the formulation of the inverse scattering as a Riemann-Hilbert problem is outlined. The solution is expressed via the scattering data in a form that admits the peakon limit in the sections that follow.

In Section 3 the map between the action-angle variables (expressed via the scattering data) and the momentum of the $\mathrm{CH}$ solution is formulated as a momentum map from the symplectic action-angle variables to the dual of the Lie algebra of smooth vector fields on the real line. This is a Poisson map, but the noncanonical bi-Hamiltonian structure of the $\mathrm{CH}$ equation which led to the discovery of its isospectral problem in [9] and its geometrical significance as geodesic flow on the diffeomorphism group are not discussed here.

In Section 4 we introduce the peakons as singular solutions that appear in dispersionless limit. The $N$-peakon solution is governed by a finite-dimensional integrable dynamical system.

Section 5 presents the multi-peakon solution as a limiting case of the $\mathrm{CH}$ multisoliton solution and points out the similarity between the dynamics of the peakon system and the well known Toda lattice.

In Section 6 we comment briefly on the existence of additional integrals of motion of the peakon system, a property known as superintegrability. The two-peakon system is analysed explicitly. 
Section 7 presents the compacton and pulson solutions, which are singular solutions, similar to peakons that can also be represented by the singular momentum map for peakons discussed in [34].

In Section 8 we deal with generalizations of the $\mathrm{CH}$ equation in higher dimensions. These also allow for singular solutions. Although such multidimensional generalizations are unlikely to be integrable, numerical studies show that their solutions are stable and interact elastically in collisions.

In Section 9 we comment briefly on some limitations of our present discussion and point out three open problems for further research.

\section{Soliton Solutions of CH Equation from Inverse Scattering}

\subsection{Inverse Scattering for the KdV Equation}

One of the most significant results in the theory of nonlinear partial differential equations was the development of Gardner, Greene, Kruskal and Miura [28, 29] of a method for the exact solution of the initial-value problem for the KdV equation. Prior to their work the only known exact solutions of KdV were the travelling wave solutions. The idea is based on a representation of the nonlinear equation $u_{t}+6 u u_{x}+u_{x x x}=0$ as a compatibility condition of two eigenvalue problems with a time-independent spectral parameter $\lambda$

$$
\Psi_{x x}+u \Psi=\lambda \Psi, \quad \Psi_{t}=\left(u_{x}+\gamma\right) \Psi-(4 \lambda+2 u) \Psi_{x}
$$

where $\gamma$ is an arbitrary constant. The method is conceptually analogous in many ways to the Fourier transform method for solving linear equations. This method is now known as IST. It was recast by Lax [42] in a general framework that allows the IST to be used for solving other nonlinear PDEs. An important consequence is the relationship between the discrete eigenvalues and the characteristics of the solitons that emerge asymptotically. Another feature of soliton collisions is the preservation of soliton identities after the interaction (asymptotically in time). For example, collisions of $\mathrm{KdV}$ solitons only result in a phase shift from the positions where they would have been without the interaction.

The KdV was formulated as a completely integrable Hamiltonian system in a work by Faddeev and Zakharov [56], the Hamiltonian form was also noted by Gardner [27].

Reviews of IST may be found, for example, in Ablowitz et al [1], Dubrovin et al [20], Novikov et al [48]. For discussions of other related bi-Hamiltonian equations, see [19]. We note that the spectrum of the corresponding eigenvalue problem, e.g. (6), depends on the boundary conditions of the solution, see [30]. 


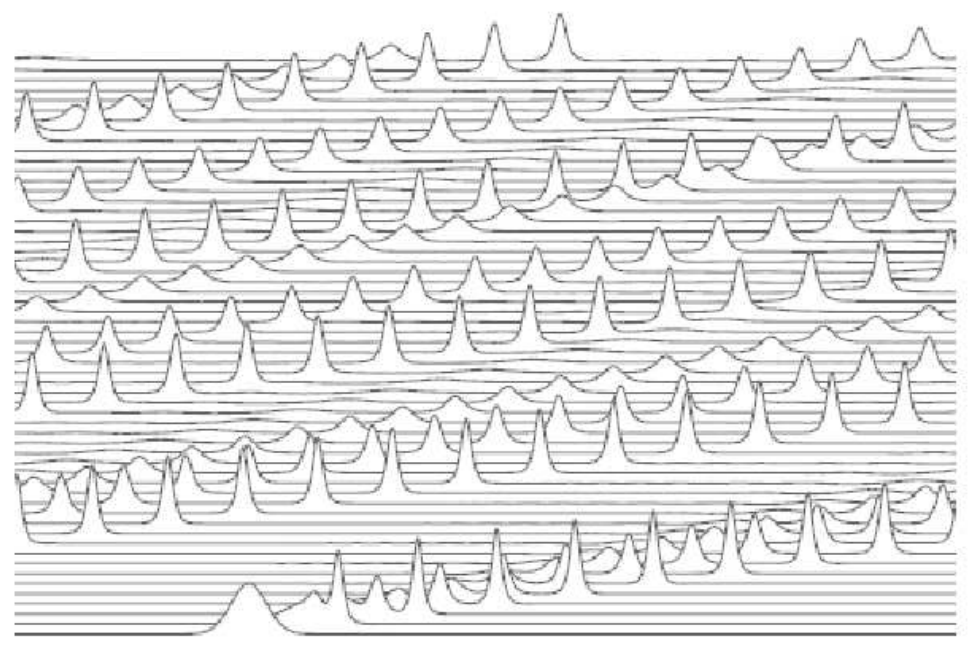

Figure 1. A Gaussian initial condition for the $\mathrm{CH}$ equation breaks up into an ordered train of solitons as time evolves (the time direction being vertical). The soliton train eventually wraps around the periodic domain, thereby allowing the leading solitons to overtake the slower emergent solitons from behind in collisions that cause phase shifts, as discussed in [9].

\subsection{Inverse Scattering for CH Solitons with Dispersion}

In this section we outline the application of the IST for the $\mathrm{CH}$ equation. We use the form with a linear dispersion term

$$
u_{t}-u_{x x t}+2 \omega u_{x}+3 u u_{x}-2 u_{x} u_{x x}-u u_{x x x}=0
$$

where $\omega$ is a real constant. This equation appears as a model of unidirectional propagation of shallow water waves over a flat bottom [9, 38, 39] as well as that of axially symmetric waves in a hyperelastic rod [18]. It can be obtained from the $b$-equation (4) with $b=2$ via Galilean transformation that removes the $u_{x x x}$ term. Equation (7) admits a Lax pair formulation [9]

$$
\begin{aligned}
\Psi_{x x} & =\frac{1}{4}(1-(m+\omega) \lambda) \Psi \\
\Psi_{t} & =-\left(\frac{2}{\lambda}+u\right) \Psi_{x}+\frac{u_{x}}{2} \Psi+\gamma \Psi
\end{aligned}
$$

where $\gamma$ is an arbitrary constant. We will use this freedom for a proper normalization of the eigenfunctions.

In our further considerations $m$ will be a Schwartz class function, $\omega>0$ and $m(x, 0)+\omega>0$. Then $m(x, t)+\omega>0$ for all $t$ [11]. Let us introduce a new 
spectral parameter $k$, such that

$$
\lambda(k)=\frac{1}{\omega}\left(1+4 k^{2}\right) .
$$

The spectrum of the problem (8) under these conditions is described in [10]. The continuous spectrum in terms of $k$ corresponds to $k$-real. The discrete spectrum (in the upper half plane) consists of finitely many points $k_{n}=\mathrm{i} \kappa_{n}, n=1, \ldots, N$ where $\kappa_{n}$ is real and $0<\kappa_{n}<1 / 2$.

For all real $k \neq 0$ a basis in the space of solutions of (8) can be introduced, $\psi(x, k)$ and $\bar{\psi}(x, \bar{k})$, fixed by its asymptotic when $x \rightarrow \infty[10]$

$$
\psi(x, k)=\mathrm{e}^{-\mathrm{i} k x}+o(1), \quad x \rightarrow \infty .
$$

Another basis can be introduced, $\varphi(x, k)$ and $\bar{\varphi}(x, \bar{k})$ fixed by its asymptotic when $x \rightarrow-\infty$

$$
\varphi(x, k)=\mathrm{e}^{-\mathrm{i} k x}+o(1), \quad x \rightarrow-\infty
$$

and the relation between the two bases is [13]

$$
\varphi(x, k)=a(k) \psi(x, k)+b(k) \bar{\psi}(x, k)
$$

where

$$
|a(k)|^{2}-|b(k)|^{2}=1
$$

The quantity $\mathcal{R}(k)=b(k) / a(k)$ is called the reflection coefficient, without any danger of confusing $b(k)$ with the bifurcation parameter $b$ introduced in (4).

All of the required information about the scattering, i.e., $a(k)$ and $b(k)$, is provided by $\mathcal{R}(k)$ for $k>0$ only [15]. It is sufficient to know $\mathcal{R}(k)$ only on the half line $k>0$, since $\bar{a}(k)=a(-k), \bar{b}(k)=b(-k)$ and thus $\mathcal{R}(-k)=\overline{\mathcal{R}}(k)$.

The constant $\gamma$ in (9) can be chosen for each eigenfunction in such a way that $a(k)$ does not depend on $t$ and is a generating function of the integrals of motion [15]. At the points of the discrete spectrum, $a(k)$ has simple zeroes [10], therefore $\varphi$ and $\bar{\psi}$ are linearly dependent (13)

$$
\varphi\left(x, \mathrm{i} \kappa_{n}\right)=b_{n} \bar{\psi}\left(x,-\mathrm{i} \kappa_{n}\right) .
$$

In other words, the discrete spectrum is simple, there is only one (real) eigenfunction $\varphi^{(n)}(x)$, corresponding to each eigenvalue $\mathrm{i} \kappa_{n}$, and we can take this eigenfunction to be

$$
\varphi^{(n)}(x) \equiv \varphi\left(x, \mathrm{i} \kappa_{n}\right)
$$


The asymptotic behaviour of $\varphi^{(n)}$, according to (12), (15) is

$$
\begin{aligned}
& \varphi^{(n)}(x)=\mathrm{e}^{\kappa_{n} x}+o\left(\mathrm{e}^{\kappa_{n} x}\right), \quad x \rightarrow-\infty \\
& \varphi^{(n)}(x)=b_{n} \mathrm{e}^{-\kappa_{n} x}+o\left(\mathrm{e}^{-\kappa_{n} x}\right), \quad x \rightarrow \infty
\end{aligned}
$$

The sign of $b_{n}$ obviously depends on the number of the zeroes of $\varphi^{(n)}$. Suppose that

$$
0<\kappa_{1}<\kappa_{2}<\ldots<\kappa_{N}<1 / 2 .
$$

Then from the oscillation theorem for the Sturm-Liouville problem [7], $\varphi^{(n)}$ has exactly $n-1$ zeroes. Therefore

$$
b_{n}=(-1)^{n-1}\left|b_{n}\right| .
$$

The set

$$
\mathcal{S} \equiv\left\{\mathcal{R}(k) ; k>0, \quad \kappa_{n}, \quad R_{n}=b_{n} / \mathrm{i} a^{\prime}\left(\mathrm{i} \kappa_{n}\right), \quad n=1, \ldots N\right\}
$$

is called the scattering data. The Hamiltonians for the $\mathrm{CH}$ equation in terms of the scattering data are presented in [15]. The time evolution of the scattering data is [13]

$$
\mathcal{R}(k, t)=\mathcal{R}(k, 0) \exp \left(-\frac{4 \mathrm{i} k}{\lambda} t\right), \quad R_{n}(t)=R_{n}(0) \exp \left(\frac{4 \kappa_{n}}{\lambda_{n}} t\right)
$$

where $R_{n}(t)$ is always a positive quantity [13] and $\lambda_{n}=\lambda\left(\kappa_{n}\right)$.

The scattering coefficient $a(k)$ is analytic for $\operatorname{Im} k>0$ with asymptotic [15]

$$
\mathrm{e}^{\mathrm{i} \beta k} a(k) \rightarrow 1, \quad|k| \rightarrow \infty
$$

where $\beta$ is a constant (integral of motion)

$$
\beta=\int_{-\infty}^{\infty}\left(\sqrt{1+\frac{m(x)}{\omega}}-1\right) \mathrm{d} x .
$$

The asymptotics of the Jost solutions (or, rather the following quantities, depending on the Jost solutions) for $|k| \rightarrow \infty$ have the form [13]

$$
\begin{aligned}
& \underline{\psi}(x, k) \equiv \psi(x, k) \mathrm{e}^{\frac{\mathrm{i} k y}{\sqrt{\omega}}}=X_{0}(x)+\frac{X_{1}(x)}{k}+\frac{X_{2}(x)}{k^{2}}+\ldots \\
& \underline{\varphi}(x, k) \equiv \varphi(x, k) \mathrm{e}^{\mathrm{i} k\left(\frac{y}{\sqrt{\omega}}+\beta\right)}=X_{0}(x)+\frac{\widetilde{X}_{1}(x)}{k}+\frac{\widetilde{X}_{2}(x)}{k^{2}}+\ldots
\end{aligned}
$$


where $X_{0}(x)=[\omega /(m(x)+\omega)]^{1 / 4}>0$ and

$$
y(x)=\sqrt{\omega}\left[x+\int_{\infty}^{x}\left(X_{0}^{-2}(\tilde{x})-1\right) \mathrm{d} \tilde{x}\right] .
$$

Moreover, (17) is analytic for $\operatorname{Im}(k)<0,(18)$ is analytic for $\operatorname{Im}(k)>0$ [13].

From (13) with (17) and (18) we obtain

$$
\frac{\varphi(x, k)}{\mathrm{e}^{\mathrm{i} k \beta} a(k)}=\underline{\psi}(x, k)+\mathcal{R}(k) \underline{\bar{\psi}}(x, k) \mathrm{e}^{2 \mathrm{i} k y(x) / \sqrt{\omega}} .
$$

The function $\varphi(x, k) /\left(\mathrm{e}^{\mathrm{i} k \beta} a(k)\right)$ is analytic for $\operatorname{Im}(k)>0, \psi(x, k)$ is analytic for $\operatorname{Im}(k)<0$. Thus, (20) represents an additive Riemann-Hilbert Problem (RHP) with a jump on the real line, given by $\mathcal{R}(k) \underline{\bar{\psi}}(x, k) \mathrm{e}^{2 \mathrm{i} k y(x) / \sqrt{\omega}}$.

The solution of this RHP follows a standard pattern, see [13] for more details. The solution simplifies considerably in the case $\mathcal{R}(k) \equiv 0$. This is the $N$-soliton solution

$$
\begin{array}{ccc}
\underline{\psi}(x, k) & =X_{0}(x)-\mathrm{i} \sum_{n=1}^{N} \frac{R_{n}(t) \mathrm{e}^{-2 \kappa_{n} y / \sqrt{\omega}} \underline{\psi}\left(x,-\mathrm{i} \kappa_{n}\right)}{\mathrm{i} \kappa_{n}-k}, & \operatorname{Im}(k)<0 \\
\frac{\varphi(x, k)}{\mathrm{e}^{\mathrm{i} k \beta} a(k)}=X_{0}(x)-\mathrm{i} \sum_{n=1}^{N} \frac{R_{n}(t) \mathrm{e}^{-2 \kappa_{n} y / \sqrt{\omega}} \underline{\psi}\left(x,-\mathrm{i} \kappa_{n}\right)}{\mathrm{i} \kappa_{n}-k}, & \operatorname{Im}(k)>0 .
\end{array}
$$

From (21) one has a linear system for the quantities $\underline{\psi}\left(x,-\mathrm{i} \kappa_{n}, t\right)$ with a solution

$$
\underline{\psi}\left(x,-\mathrm{i} \kappa_{n}, t\right)=X_{0}(x) \sum_{p=1}^{N} A_{n p}^{-1}[y, t], \quad n=1, \ldots, N
$$

where

$$
A_{p n}[y, t] \equiv \delta_{p n}+\frac{R_{n}(t) \mathrm{e}^{-2 \kappa_{n} y / \sqrt{\omega}}}{\kappa_{p}+\kappa_{n}} .
$$

Taking $k=-\mathrm{i} / 2$ in (21) with (23) we have

$$
\mathrm{e}^{-\frac{1}{2}\left(x-\frac{y}{\sqrt{\omega}}\right)} \equiv \underline{\psi}(x,-\mathrm{i} / 2)=X_{0}(x)\left(1-\sum_{n, p=1}^{N} \frac{R_{n}(t) \mathrm{e}^{-2 \kappa_{n} y / \sqrt{\omega}}}{\kappa_{n}+\frac{1}{2}} A_{n p}^{-1}[y, t]\right) .
$$


The substitution $\kappa=\mathrm{i} / 2$ in (22) with $a(\mathrm{i} / 2)=1$ gives

$$
\mathrm{e}^{\frac{1}{2}\left(x-\frac{y}{\sqrt{\omega}}\right)} \equiv \frac{\underline{\varphi}(x, \mathrm{i} / 2)}{\mathrm{e}^{-\beta / 2} a(\mathrm{i} / 2)}=X_{0}(x)\left(1-\sum_{n, p=1}^{N} \frac{R_{n}(t) \mathrm{e}^{-2 \kappa_{n} y / \sqrt{\omega}}}{\kappa_{n}-\frac{1}{2}} A_{n p}^{-1}[y, t]\right) .
$$

From (25) and (26) there follows a parametric representation

$$
\begin{aligned}
x & =X(y, t) \equiv \frac{y}{\sqrt{\omega}}+\ln \frac{f_{+}}{f_{-}} \\
f_{ \pm} & \equiv 1-\sum_{n, p=1}^{N} \frac{R_{n}(t) \mathrm{e}^{-2 \kappa_{n} y / \sqrt{\omega}}}{\kappa_{n} \mp \frac{1}{2}} A_{n p}^{-1}[y, t] .
\end{aligned}
$$

\subsection{Parametric Form of the Dispersive CH Soliton Solution}

From (19) and (27) one can compute the solution in parametric form

$$
u(X(y, t), t)=X_{t}(y, t), \quad x=X(y, t)
$$

where $X(y, t)$ is given in terms of the scattering data in (27), (28). Upon introducing new notations

$$
\begin{aligned}
\xi_{j} & =2 \kappa_{j}\left(-\frac{y}{\sqrt{\omega}}+\frac{2 t}{\lambda_{j}}+x_{j 0}\right) \\
x_{j 0} & =\frac{1}{2 \kappa_{j}} \ln \frac{R_{j}(0)}{2 \kappa_{j}} \\
\phi_{j} & =\ln \frac{1-2 \kappa_{j}}{1+2 \kappa_{j}} \\
\gamma_{i j} & =\ln \left(\frac{\kappa_{i}-\kappa_{j}}{\kappa_{i}+\kappa_{j}}\right)^{2}
\end{aligned}
$$

one can rewrite the expression for $f_{ \pm}(28)$ in the form $[45,49-51]$

$$
f_{ \pm} \equiv \sum_{\mu=0,1} \exp \left[\sum_{i=1}^{N} \mu_{i}\left(\xi_{i} \mp \phi_{i}\right)+\sum_{1 \leq i<j \leq j} \mu_{i} \mu_{j} \gamma_{i j}\right]
$$

The solution for $m$ can be obtained from (29). First we notice that

$$
\frac{\partial u(X(y, t), t)}{\partial y}=u_{x}(X(y, t), t) X_{y}
$$


and also

$$
\frac{\partial u(X(y, t), t)}{\partial y}=\frac{\partial X_{t}}{\partial y}=X_{t y}
$$

Thus $u_{x}(X(y, t), t)=X_{t y} / X_{y}$. Similarly

$$
u_{x x}(X(y, t), t)=\frac{1}{X_{y}}\left(\frac{X_{t y}}{X_{y}}\right)_{y}
$$

and

$$
m(X(t, y), t)=u(X(t, y), t)-u_{x x}(X(t, y), t)=X_{t}-\frac{1}{X_{y}}\left(\frac{X_{t y}}{X_{y}}\right)_{y}
$$

with

$$
\begin{aligned}
m(x, t) & =\int_{-\infty}^{\infty} P(y, t) \delta(x-X(y, t)) \mathrm{d} y \\
P(y, t) & =X_{t} X_{y}-\left(\frac{X_{t y}}{X_{y}}\right)_{y} \\
u(x, t) & =\frac{1}{2} \int_{-\infty}^{\infty} P(y, t) \exp (-|x-X(y, t)|) \mathrm{d} y .
\end{aligned}
$$

As we shall see in Section 8, this representation is also useful in the study of multidimensional solutions.

\subsection{Relation to KdV Hierarchy}

The spectral problem (8) is gauge equivalent to a standard Sturm-Liouville problem, well known from the KdV hierarchy, cf. (6), with short notation $q=m+\omega$ :

$$
\begin{aligned}
-\Phi_{y y}+U(y) \Phi & =\mu \Phi, \quad \mu=\frac{\lambda}{4}-\frac{1}{4 \omega} \\
\Phi(y) & =q^{1 / 4} \Psi, \quad \frac{\mathrm{d} y}{\mathrm{~d} x}=\sqrt{q} \\
U(y) & =\frac{1}{4 q(y)}+\frac{q_{y y}(y)}{4 q(y)}-\frac{3 q_{y}^{2}(y)}{16 q^{2}(y)}-\frac{1}{4 \omega}
\end{aligned}
$$

Note that (32) leads to two possible expressions for the change of the variables in the Liouville transformation

$$
\begin{aligned}
& y=\sqrt{\omega} x+\int_{-\infty}^{x}\left(\sqrt{q\left(x^{\prime}\right)}-\sqrt{\omega}\right) \mathrm{d} x^{\prime}+\text { const } \\
& y=\sqrt{\omega} x+\int_{\infty}^{x}\left(\sqrt{q\left(x^{\prime}\right)}-\sqrt{\omega}\right) \mathrm{d} x^{\prime}+\text { const } .
\end{aligned}
$$


These two possibilities are only consistent iff

$$
\int_{-\infty}^{\infty}(\sqrt{q(x)}-\sqrt{\omega}) \mathrm{d} x=\text { const }
$$

which is always the case, since the integral under question is (up to a multiplier) the Casimir function $\beta$ (16).

The matching of the $\mathrm{CH}$ hierarchy to $\mathrm{KdV}$ hierarchy requires solving the ErmakovPinney equation (32) [10], which is not straightforward and leads to the same solution (29) in parametric form.

\section{Momentum Map Formulation with Action-angle Variables}

The canonical Poisson brackets for the scattering data of the $\mathrm{CH}$ equation are computed in [15] where also the action-angle variables are expressed in terms of the scattering data. Let us consider the action variable for the N-soliton solution. (These considerations can be extended easily to the variables of the continuous spectrum.) The angle variable is $\Phi_{n}=\ln R_{n}(t)$ and it is linear in time $t$ and $\dot{\Phi}_{n}=4 \kappa_{n} / \lambda_{n}$. Let us introduce $\Lambda_{n}:=4 \kappa_{n} / \lambda_{n}$ into Hamilton's principle $\delta S=0$, with

$$
S\left[u, \Phi_{n}, \Pi_{n}\right]=\int\left(\ell[u]+\sum_{n=1}^{N} \Pi_{n}\left(\dot{\Phi}_{n}-\Lambda_{n}[u]\right)\right) \mathrm{d} t .
$$

Here the Lagrange multiplier $\Pi_{n}$ enforces the action-angle relation for the $\mathrm{CH}$ scattering data as a $\mathbb{T}^{N}$ shift of the angles $\Phi_{n}$ at constant angular frequencies $\Lambda_{n}$, with $n=1, \ldots, N$. The stationary variation is

$$
\delta S=\int\left(\left(\frac{\delta \ell}{\delta u}-\sum_{n=1}^{N} \Pi_{n} \frac{\delta \Lambda_{n}}{\delta u}\right) \delta u+\sum_{n=1}^{N}\left(\dot{\Phi}_{n}-\Lambda_{n}[u]\right) \delta \Pi_{n}-\sum_{n=1}^{N} \dot{\Pi}_{n} \delta \Phi_{n}\right) \mathrm{d} t
$$

Since by definition $m=\delta \ell / \delta u$ is the momentum, $\delta S=0$ implies the Eulerian representation

$$
\begin{aligned}
& m=\frac{\delta \ell}{\delta u}=\sum_{n=1}^{N} \Pi_{n} \frac{\delta \Lambda_{n}}{\delta u}, \quad \text { with } \\
& \dot{\Phi}_{n}=\Lambda_{n}[u] \quad \text { and } \quad \dot{\Pi}_{n}=0 .
\end{aligned}
$$

Relation (33) is the momentum map

$$
\left(\Phi_{n}, \Pi_{n}\right) \in T^{*} \mathbb{T}^{N} \rightarrow m \in \mathfrak{X}^{*}
$$


for the toral $\mathbb{T}^{N}$ action (34) on the angles $\Phi_{n}$ at constant angular frequencies $\Lambda_{n}$. This momentum map from the action-angle scattering variables $T^{*}\left(\mathbb{T}^{N}\right)$ to the flow momentum $\mathfrak{X}^{*}(\mathbb{R})$ (dual to the smooth vector fields $\mathfrak{X}(\mathbb{R})$ on the real line) provides the Eulerian representation of the $\mathrm{N}$-soliton solution of $\mathrm{CH}$ in terms of the scattering data and squared eigenfunctions of its isospectral eigenvalue problem. Momentum maps for Hamiltonian dynamics are reviewed in [44], for example.

By using the spectral quantities of the $N$-soliton solution and recalling that $\lambda_{n}=$ $\left(1-4 \kappa_{n}^{2}\right) / \omega$ one may express the variation of the spectrum with respect to the $\mathrm{CH}$ solution in terms of the squared-eigenfunctions of the isospectral problem as [15]

$$
\frac{\delta \Lambda_{n}}{\delta m(x, t)}=\frac{\left(1+4 \kappa_{n}^{2}\right)}{2 \omega \kappa_{n} \lambda_{n}} R_{n}(t)\left[\bar{\psi}\left(x,-\mathrm{i} \kappa_{n}, t\right)\right]^{2}
$$

in which $\bar{\psi}\left(x,-\mathrm{i} \kappa_{n}, t\right)$ is the eigenfunction that belongs to eigenvalue $\lambda_{n}$, see (15). On the other hand, the expansion of $u(x, t)$ over squares of eigenfunctions is given by [14]

$$
u(x, t)=\sum_{n=1}^{N} \frac{4 \kappa_{n}}{\omega \lambda_{n}^{2}} R_{n}(t)\left[\bar{\psi}\left(x,-\mathrm{i} \kappa_{n}, t\right)\right]^{2} .
$$

Consequently

$$
m(x, t)=\sum_{n=1}^{N} \frac{4 \kappa_{n}}{\omega \lambda_{n}^{2}} R_{n}(t)\left(1-\partial^{2}\right)\left[\bar{\psi}\left(x,-\mathrm{i} \kappa_{n}, t\right)\right]^{2}
$$

or

$$
m(x, t)=\sum_{n=1}^{N} \Pi_{n} J_{n}(x, t)
$$

where $\Pi_{n}$ and $J_{n}(x, t)$ denote explicitly

$$
\begin{aligned}
\Pi_{n} & =\frac{8 \kappa_{n}^{2}}{\lambda_{n}\left(1+4 \kappa_{n}^{2}\right)}=\frac{2 \Lambda_{n} \kappa_{n}}{1+4 \kappa_{n}^{2}} \\
J_{n}(x, t) & \equiv \frac{\delta \Lambda_{n}}{\delta u(x, t)}=\frac{\left(1+4 \kappa_{n}^{2}\right)}{2 \omega \kappa_{n} \lambda_{n}} R_{n}(t)\left(1-\partial^{2}\right)\left[\bar{\psi}\left(x,-\mathrm{i} \kappa_{n}, t\right)\right]^{2} .
\end{aligned}
$$

Thus, the momentum map (33) from the action-angle variables under going dynamics (34) to the Eulerian representation of the momentum for the $\mathrm{CH}$ solution is expressed in terms of the scattering data and squared eigenfunctions of its $\mathrm{N}$ soliton isospectral eigenvalue problem. Perhaps not unexpectedly, this momentum map may be applied to the action-angle representation of the solution of any integrable Hamiltonian PDE. 


\section{Peakons}

\subsection{Peakons: the Singular Solution Ansatz}

Camassa and Holm [9] discovered the "peakon" solitary traveling wave solution for a shallow water wave

$$
u(x, t)=c \mathrm{e}^{-|x-c t| / \alpha}
$$

whose fluid velocity $u$ is a function of position $x$ on the real line and time $t$. The peakon traveling wave moves at a speed equal to its maximum height, at which it has a sharp peak (jump in derivative). Peakons are an emergent phenomenon, solving the initial value problem for a PDE derived by an asymptotic expansion of Euler's equations using the small parameters of shallow water dynamics. Peakons are nonanalytic solitons, which superpose as

$$
\left.u(x, t)=\frac{1}{2} \sum_{a=1}^{N} p_{a}(t) \mathrm{e}^{-\left|x-q_{a}(t)\right| / \alpha}=: \frac{1}{2} \sum_{a=1}^{N} p_{a}(t) g\left(x-q_{a}(t)\right) / \alpha\right)
$$

for sets $\{p\}$ and $\{q\}$ satisfying canonical Hamiltonian dynamics. Peakons arise for shallow water waves in the limit of zero linear dispersion in one dimension. Peakons satisfy a PDE arising from Hamilton's principle for geodesic motion on the smooth invertible maps (diffeomorphisms) with respect to the $H^{1}$ Sobolev norm of the fluid velocity. Peakons generalize to higher dimensions, as well. We explain how peakons were derived in the context of shallow water asymptotics and describe some of their remarkable mathematical properties.

Peakons were first found as singular soliton solutions of the completely integrable $\mathrm{CH}$ equation. This is equation (4) with $b=2$, now rewritten in terms of the velocity, as

$$
u_{t}+c_{0} u_{x}+3 u u_{x}+\Gamma u_{x x x}=\alpha^{2}\left(u_{x x t}+2 u_{x} u_{x x}+u u_{x x x}\right) .
$$

Peakons were found in [9] to arise in the absence of linear dispersion. That is, they arise when $c_{0}=0$ and $\Gamma=0$ in $\mathrm{CH}$ (36). Specifically, peakons are the individual terms in the peaked $N$-soliton solution of $\mathrm{CH}$ (36) for its velocity, in the absence of linear dispersion. Each term in the sum (35) is a solition with a sharp peak at its maximum. Hence, the name "peakon." Expressed using its momentum, $m=\left(1-\alpha^{2} \partial_{x}^{2}\right) u$, the peakon velocity solution (35) of dispersionless $\mathrm{CH}$ becomes a sum over a delta functions, supported on a set of points moving on the real line. Namely, the peakon velocity solution (35) implies

$$
m(x, t)=\alpha \sum_{a=1}^{N} p_{a}(t) \delta\left(x-q_{a}(t)\right)
$$


because of the relation $\left(1-\alpha^{2} \partial_{x}^{2}\right) \mathrm{e}^{-|x| / \alpha}=2 \alpha \delta(x)$. These solutions satisfy the $b$-equation (4) for any value of $b$, provided $c_{0}=0$ and $\Gamma=0$. As we shall discuss later, the peakon momentum relation (37) is again a momentum map.

Thus, peakons are singular momentum solutions of the dispersionless $b$-equation, although they are not stable for every value of $b$. From numerical simulations [36], peakons are conjectured to be stable for $b>1$. In the integrable cases $b=2$ for $\mathrm{CH}$ and $b=3$ for DP, peakons are stable singular soliton solutions. The spatial velocity profile $\mathrm{e}^{-|x| / \alpha} /(2 \alpha)$ of each separate peakon in (35) is the Green's function for the Helmholtz operator on the real line, with vanishing boundary conditions at spatial infinity. Unlike the KdV soliton, whose speed and width are related, the width of the peakon profile is set by its Green's function, independently of its speed.

\subsection{Integrable Peakon Dynamics of $\mathrm{CH}$}

Substituting the peakon solution ansatz (35) and (37) into the dispersionless $\mathrm{CH}$ equation,

$$
m_{t}+u m_{x}+2 m u_{x}=0, \quad \text { with } \quad m=u-\alpha^{2} u_{x x}
$$

yields Hamilton's canonical equations for the dynamics of the discrete set of peakon parameters $p_{a}(t)$ and $q_{a}(t)$

$$
\dot{q}_{a}(t)=\frac{\partial h_{N}}{\partial p_{a}} \quad \text { and } \quad \dot{p}_{a}(t)=-\frac{\partial h_{N}}{\partial q_{a}}
$$

for $a=1,2, \ldots, N$, with Hamiltonian [9] given by

$$
h_{N}=\frac{1}{4} \sum_{a, b=1}^{N} p_{a} p_{b} \mathrm{e}^{-\left|q_{a}-q_{b}\right| / \alpha} .
$$

Or explicitly

$$
\begin{aligned}
& \dot{q}_{a}=\frac{1}{2} \sum_{b=1}^{N} p_{b} \mathrm{e}^{-\left|q_{a}-q_{b}\right| / \alpha} \\
& \dot{p}_{a}=\frac{p_{a}}{2 \alpha} \sum_{b=1}^{N} p_{b} \mathrm{e}^{-\left|q_{a}-q_{b}\right| / \alpha} \operatorname{sgn}\left(q_{a}-q_{b}\right) .
\end{aligned}
$$

Thus, one finds that the points $x=q_{a}(t)$ in the peakon solution (35) move with the flow of the fluid velocity $u$ at those points, since $u\left(q_{a}(t), t\right)=\dot{q}_{a}(t)$. This means the $q_{a}(t)$ are Lagrangian coordinates. Moreover, the singular momentum solution (37) is the Lagrange-to-Euler map for an invariant manifold of the dispersionless 
$\mathrm{CH}$ equation (38). On this finite-dimensional invariant manifold for the PDE (38), the dynamics is canonically Hamiltonian.

With Hamiltonian (40), the canonical equations (39) for the $2 N$ canonically conjugate peakon parameters $p_{a}(t)$ and $q_{a}(t)$ were interpreted in [9] as describing geodesic motion on the $N$-dimensional Riemannian manifold whose co-metric is $g^{a b}(\{q\})=\mathrm{e}^{-\left|q_{a}-q_{b}\right| / \alpha}$. Moreover, the canonical geodesic equations arising from this Hamiltonian comprise an integrable system for any number of peakons $N$. This integrable system was studied in [9] for solutions on the real line, and in $[3,16]$ and references therein, for spatially periodic solutions.

The integrals generated by the action variables in terms of the coordinates can be recovered as $\operatorname{tr}\left(L^{k}\right)$, where $L$ is the Lax operator for the peakon system [9].

Being a completely integrable Hamiltonian soliton equation, the continuum $\mathrm{CH}$ equation (36) has an associated isospectral eigenvalue problem, discovered in [9] for any values of its dispersion parameters $c_{0}$ and $\Gamma$. Remarkably, when $c_{0}=0$ and $\Gamma=0$, this isospectral eigenvalue problem has a purely discrete spectrum. Moreover, in this case, each discrete eigenvalue corresponds precisely to the timeasymptotic velocity of a peakon. This discreteness of the $\mathrm{CH}$ isospectrum in the absence of linear dispersion implies that only the singular peakon solutions (37) emerge asymptotically in time, in the solution of the initial value problem for the dispersionless $\mathrm{CH}$ equation (38). This is borne out in numerical simulations of the dispersionless $\mathrm{CH}$ equation (38), starting from a smooth initial distribution of velocity $[26,36]$.

Figure 2 shows the emergence of peakons from an initially Gaussian velocity distribution and their subsequent elastic collisions in a periodic one-dimensional domain. This figure demonstrates that singular solutions dominate the initial value problem and, thus, that it is imperative to go beyond smooth solutions for the $\mathrm{CH}$ equation; the situation is similar for the EPDiff equation.

\section{Peakons as Mechanical Systems}

Governed by canonical Hamiltonian equations, each $N$-peakon solution can be associated with a mechanical system of moving particles. Calogero et al [8] further extended the class of mechanical systems of this type. The r-matrix approach was applied to the Lax pair formulation of the $N$-peakon system for $\mathrm{CH}$ by Ragnisco and Bruschi [53], who also pointed out the connection of this system with the classical Toda lattice. A discrete version of the Adler-Kostant-Symes factorization method was used by Suris [54] to study a discretization of the peakon lattice, realized as a discrete integrable system on a certain Poisson submanifold of $\mathfrak{g l}(N)$ 


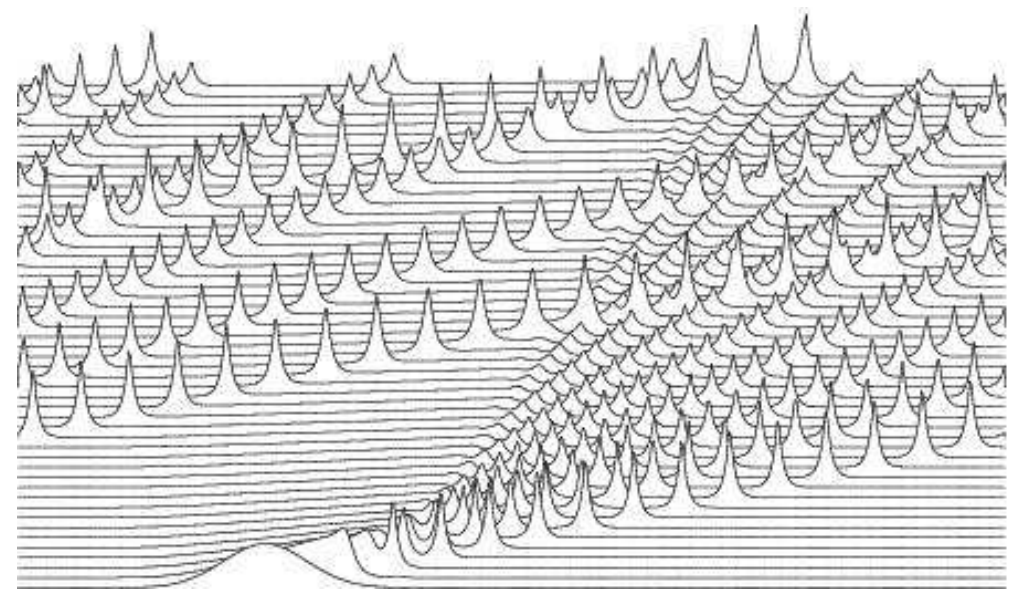

Figure 2. A Gaussian initial condition for the $\mathrm{CH}$ equation breaks up into an ordered train of peakons as time evolves (the time direction being vertical). The peakon train eventually wraps around the periodic domain, thereby allowing the leading peakons to overtake the slower emergent peakons from behind in collisions that cause phase shifts, as discussed in [9].

equipped with an $r$-matrix Poisson bracket. Beals et al [6] used the Stieltjes theorem on continued fractions and the classical moment problem for studying multipeakon solutions of the $\mathrm{CH}$ equation. Generalized peakon systems are described for any simple Lie algebra by Alber et al [3].

\section{Peakon Limit of the CH Soliton Solutions}

The limit $\omega \rightarrow 0$ in the $N$-soliton solution $u(x, t)$ produces the $N$-peakon solution (35). The limiting procedure is described in detail in [46]. Due to (10) one can write for the discrete eigenvalues $k_{n}=\mathrm{i} \kappa_{n}$

$$
2 \kappa_{j}=\left(1-\omega \lambda_{j}\right)^{1 / 2}=1-\frac{1}{2} \omega \lambda_{j}+\ldots
$$

The solution (27) depends explicitly on $\kappa_{j}$ (43) and the limit can be computed with (43) by taking $\omega \rightarrow 0$ and keeping the eigenvalue $\lambda_{j}$ constant. The result is the 
expression (35) with

$$
\begin{array}{ll}
p_{i}=\frac{4 D_{N-i+1}^{(0)} D_{N-i}^{(2)}}{D_{N-i+1}^{(1)} D_{N-i}^{(1)}}, & i=1,2, \ldots, N \\
q_{i}=\alpha \ln \left[\frac{2 D_{N-i+1}^{(0)}}{D_{N-i}^{(2)}}\right], & i=1,2, \ldots, N
\end{array}
$$

where

$$
\begin{gathered}
D_{n}^{(m)}=\sum_{1 \leq i_{1}<i_{2}<\ldots<i_{n} \leq N} \Delta_{n}\left(i_{1}, i_{2}, \ldots, i_{n}\right)\left(\lambda_{i_{1}} \lambda_{i_{2}} \ldots \lambda_{i_{n}}\right)^{m} R_{i_{1}} R_{i_{2}} \ldots R_{i_{n}} \\
\Delta_{n}\left(i_{1}, i_{2}, \ldots, i_{n}\right)=\prod_{1 \leq l<m \leq n}\left(\lambda_{i_{l}}-\lambda_{i_{m}}\right)^{2}, \quad n \geq 2 \\
R_{i}(t)=R_{i}(0) e^{\frac{2}{\lambda_{i}} t}, \quad x_{i 0}=\ln R_{i}(0) .
\end{gathered}
$$

Usually the quantities $D_{n}^{(m)}$ are called Hankel determinants. By definition $D_{0}^{(m)}=1$. In general, Hankel determinant is a determinant of $n \times n$ matrix of the form $D_{n}^{(m)} \equiv \operatorname{det}\left(a_{i j}^{(m)}\right)$ where $a_{i j}$ are elements of a sequence, i.e.,

$$
a_{i j}^{(m)}=A_{i+j+m-2}
$$

In this particular case

$$
A_{l}=\sum_{i=1}^{N} \lambda_{i}^{l} R_{i}(t) .
$$

\section{Mixture of Peakons and 'Anti-peakons'}

The peakon solution (44) was obtained from the soliton solution under the assumption $m(x, 0)+\omega>0$. Thus, all $p_{k}$ are of the same sign, since all the eigenvalues $\lambda_{n}$ in this case are positive. However, one can formally use the same solution with eigenvalues of various signs to model $p_{k}$ of various signs (mixture of peakons and 'anti-peakons') and thus to study peakon - anti-peakon interactions, see e.g. [52]. The result is that the multi-peakon interaction (including anti-peakons) in general decomposes into a sequence of pairwise collisions [26]. The collision of a single peakon-anti-peakon pair was studied already in [9]. When the eigenvalues are of mixed signs, the Hankel determinants in the denominator of (44) may develop singularities for finite values of $t$. This 'peakon-breaking' phenomenon is apparently the analog of the wave-breaking mentioned earlier when $\omega \rightarrow 0$. 
Matsuno's form of the solution with Hankel determinants is particularly useful for the study of this phenomenon. Supposed that $i$-th and $(i+1)$-st particles are peakon and anti-peakon, i.e. $q_{i}(t)$ and $q_{i+1}(t)$ have different signs before the impact. Using the identities for the Hankel determinants

$$
\begin{aligned}
D_{n+2}^{(m)} D_{n}^{(m+2)} & =D_{n+1}^{(m+2)} D_{n+1}^{(m)}-\left(D_{n+1}^{(m+1)}\right)^{2} \\
D_{N-n+1, t}^{(2)} D_{N-n}^{(2)} & -D_{N-n+1}^{(2)} D_{N-n, t}^{(2)}=2 D_{N-n+1}^{(1)} D_{N-n}^{(3)} \\
D_{N-n+2, t}^{(0)} D_{N-n+1}^{(0)} & -D_{N-n+2}^{(0)} D_{N-n+1, t}^{(0)}=-2 D_{N-n+2}^{(-1)} D_{N-n+1}^{(1)}
\end{aligned}
$$

we find that when the $i$-th and $i+1$-st particles collide, i.e., $q_{i}(t)=q_{i+1}(t)$, $D_{N-i}^{(1)}=0$, which gives $\left|p_{i}\right|=\infty,\left|p_{i+1}\right|=\infty$. In other words, we have a 'peakon breaking' at the time of impact. Moreover, it is clear that both $p_{i}(t)$ and $p_{i+1}(t)$ change signs, i.e., jump from $-\infty$ to $+\infty$ at the moment of impact (and vice versa). This means that the peakon becomes antipeakon after the impact and vice versa. Moreover, when $D_{N-i}^{(1)}=0$, the above identities applied to the solution give $\frac{\mathrm{d}}{\mathrm{d} t}\left(q_{i}(t)-q_{i+1}(t)\right)=0$, i.e., the $i$-th and $(i+1)$-st particles are at rest relative to each other at the time of the impact. In fact, using the identities we can compute

$$
q_{i}-q_{i+1}=\ln \left(1-\frac{\left(D_{N-i}^{(1)}\right)^{2}}{D_{N-i}^{(0)} D_{N-i}^{(2)}}\right) .
$$

Since $D_{N-i}^{(0)}$ and $D_{N-i}^{(2)}$ are always positive, $q_{i}-q_{i+1} \leq 0$ with equality at the time of impact, when $D_{N-i}^{(1)}=0$. Thus, at impact the particles do not go through each other but first stop and change the direction of their motion (relative to each other).

\section{Similarity of Peakon Lattice and Toda Lattice}

Hankel determinants appear in the solutions of other integrable systems, e.g. the Toda lattice [32]. The Toda equation [55]

$$
\begin{aligned}
\frac{\mathrm{d} p_{n}}{\mathrm{~d} t} & =\mathrm{e}^{q_{n+1}-q_{n}}-\mathrm{e}^{q_{n}-q_{n-1}}, \quad n \in \mathbb{Z} \\
\frac{\mathrm{d} q_{n}}{\mathrm{~d} t} & =p_{n}
\end{aligned}
$$

is one of the most important integrable systems. We have a finite chain with $N$ nodes under the fixed ends boundary conditions $q_{0}=-q_{N+1}=\infty$. The solution 
is in the form

$$
\begin{aligned}
& q_{n}(t)=q_{1}(0)+\ln \frac{D_{n}^{(0)}}{D_{n-1}^{(0)}} \\
& q_{1}(0)=-\frac{1}{N} \ln D_{N}^{(0)}(0) \quad \text { is a const. }
\end{aligned}
$$

The Hankel determinants are obtained by a similar sequence

$$
\begin{aligned}
A_{l} & =\sum_{i=1}^{N} \lambda_{i}^{l} R_{i}(t) \\
R_{i}(t) & =R_{i}(0) \mathrm{e}^{-\lambda_{i} t}
\end{aligned}
$$

where $\lambda_{i}$ are $N$ different constants (eigenvalues of the Lax matrix) and the quantities $R_{i}(0), i=1, \ldots, N$ represent another set $N$ of constants.

Due to its simple form, the $N$-peakon solution can be used as an approximation of the $N$-soliton $\mathrm{CH}$ solution when the dispersion term is small (and the term $2 \omega u_{x}$ can be neglected). Similarly, the Toda chain with complex dynamical variables (the so-called Complex Toda Chain - CTC) provides an approximation for the Nsoliton solution of the Nonlinear Schröedinger Equation (NLS)

$$
\mathrm{i} u_{t}+\frac{1}{2} u_{x x}+|u|^{2} u=0
$$

see [31-33] for more details. Such an approximation is called adiabatic approximation and means that the $N$-soliton solution consists of $N$ well separated solitons

$$
u(x, t) \approx \sum_{k=1}^{N} \frac{2 \nu_{k} \mathrm{e}^{\mathrm{i}\left[2 \mu_{k}\left(x-\xi_{k}(t)+\delta_{k}(t)\right]\right.}}{\cosh \left(2 \nu_{k}\left(x-\xi_{k}(t)\right)\right.}
$$

i.e., the overlap between the solitons is small. The variables $q_{n}(t)$ of the CTC are related to the NLS solitons parameters by

$$
q_{k}(t)=-2 \nu_{0} \xi_{k}(t)+\mathrm{i}\left(2 \mu_{0} \xi_{k}(t)-\delta_{k}(t)\right)+\text { const }
$$

where $\xi_{k}, \delta_{k}, \mu_{k}=\frac{1}{2} \dot{\xi}_{k}$ and $\nu_{k}=\left(\frac{1}{2} \dot{\delta}_{k}-\mu_{k}^{2}\right)^{1 / 2}$ characterize the center-of-mass position, the phase, velocity and amplitude respectively of the $k$-th soliton in the chain while $\nu_{0}$ and $\mu_{0}$ are the average amplitude and velocity of the soliton train. The parameters $\xi_{k}$ and $\delta_{k}$ can be obtained as the real and imaginary parts of $q_{k}(t)$. Such soliton trains and their asymptotic behavior appear to be important for the needs of soliton based fiber optics communications. 


\section{Superintegrability of the Peakon System}

Suppose we have an integrable system with $2 N$ - dimensional phase space which in terms of the Action-Angle (canonical) variables can be represented as

$$
\dot{\Lambda}_{n}=0, \quad \dot{\Phi}_{n}=\Lambda_{n}, \quad n=1,2, \ldots, N
$$

or, if there exists a bracket such that ${ }^{1}$

$$
\left\{\Phi_{n}, \Lambda_{l}\right\}=\frac{1}{2} \delta_{n l}, \quad\left\{\Phi_{n}, \Phi_{l}\right\}=\left\{\Lambda_{n}, \Lambda_{l}\right\}=0
$$

the system is Hamiltonian with a Hamiltonian

$$
h_{N}=\Lambda_{1}^{2}+\ldots \Lambda_{N}^{2} .
$$

The integrals $\Lambda_{n}, n=1,2, \ldots, N$ are clearly in involution, which guarantees the integrability of the system. There is however another set of integrals

$$
\begin{array}{r}
I_{j}=\left(\Phi_{j}-\Phi_{j+1}\right)\left(\Lambda_{1}+\ldots+\Lambda_{N}\right)-\left(\Lambda_{j}-\Lambda_{j+1}\right)\left(\Phi_{1}+\ldots \Phi_{N}\right) \\
j=1,2, \ldots, N-1 .
\end{array}
$$

If the 'action' variables $\Lambda_{n}$ are all different, the set (49) is functionally independent from the set $\Lambda_{n}, n=1,2, \ldots, N$. In addition, the integrals (49) form another set of $N$-integrals in involution together with $H$. Due to the existence of two sets of functionally independent integrals in involution such systems are termed superintegrable.

An example of such system is Toda lattice, see the discussion in [2]. The peakon system is also superintegrable. The canonical variables in terms of the scattering data for $\mathrm{CH}$ equation can be used in the peakon limit: $\Lambda_{n}=2 / \lambda_{n}, \Phi_{n}=\ln R_{n}(t)$, the Hamiltonian (47) is also a peakon limit $(\omega \rightarrow 0)$ of the $N$-soliton Hamiltonian [15]

$$
H_{N}(\omega)=\omega^{2} \sum_{n=1}^{N}\left(\ln \frac{1-2 \kappa_{n}}{1+2 \kappa_{n}}+\frac{4 \kappa_{n}\left(1+4 \kappa_{n}^{2}\right)}{\left(1-4 \kappa_{n}^{2}\right)^{2}}\right) .
$$

The Poisson bracket for the $\mathrm{CH}$ peakon solution is

$$
\{A, B\} \equiv-\int_{-\infty}^{\infty} \frac{\delta A}{\delta m}(m \partial+\partial m) \frac{\delta B}{\delta m} \mathrm{~d} x
$$

\footnotetext{
${ }^{1}$ The $1 / 2$ coefficient in the definition of the bracket appears in order to match it to the Poisson bracket used in the $\mathrm{CH}$ Hamiltonian formulation.
} 
and the scattering data satisfy (46) with respect to (49), see [15] for the details. More interesting are the integrals (49). From (45) and (44) one can recover these integrals in coordinate form. For example, when $N=2$ we have

$$
I_{1}=\ln \frac{\sqrt{J}+p_{1}-p_{2}}{\sqrt{J}-p_{1}+p_{2}}+\frac{\sqrt{J}}{p_{1}+p_{2}}\left(\frac{q_{1}+q_{2}}{\alpha}+\ln \frac{p_{1}}{p_{2}}\right)
$$

where

$$
J=\left(p_{1}-p_{2}\right)^{2}+4 p_{1} p_{2} \mathrm{e}^{-\left|q_{1}-q_{2}\right| / \alpha} .
$$

Note that $I_{1}$ depends on both combinations $q_{1}+q_{2}$ and $q_{1}-q_{2}$ as well as the momentum variables. The Hamiltonian $h_{2}$ (which depends only on $q_{1}-q_{2}$ and the momentum variables) and $I_{1}$ form a complete system of integrals in involution. The integration of the 2-peakon system with these integrals can be performed as follows. First, one can express $q_{1}$ and $q_{2}$ in terms of $I_{1}, h_{2}$ and the momentum variables: $q_{i}=q_{i}\left(p_{1}, p_{2}, I_{1}, h_{2}\right)$. Next

$$
\dot{q}_{i}=\frac{\partial q_{i}}{\partial p_{1}} \dot{p}_{1}+\frac{\partial q_{i}}{\partial p_{2}} \dot{p}_{2} .
$$

The substitution of $\dot{q}_{i}, \dot{p}_{i}$ from (41) (42) to (50) produces an algebraic equation that gives, say $p_{2}$ as a function of $p_{1}$. Then (42) is an ODE for $p_{1}$ of the form $\dot{p}_{1}=f\left(p_{1}, I_{1}, h_{2}\right)$. Clearly, from practical viewpoint it is much more convenient to work with the other system of integrals in involution: $h_{2}$ and the conserved momentum $P=p_{1}+p_{2}$. Note that $J=4 h_{2}-3 P^{2}$ is itself an integral.

\section{Other Singular Solutions: the Dispersionless $b$-equation}

\section{Pulsons: Generalizing the Peakon Solutions of the Dispersionless $b$-equation for Other Green's Functions.}

The Hamiltonian $h_{N}$ in equation (40) depends on the Green's function for the relation between velocity $u$ and momentum $m$. However, the singular momentum solution ansatz (37) is independent of this Green's function. Thus, as discovered in Fringer and Holm [26], one can state

The singular momentum solution ansatz (37) for the dispersionless equation,

$$
m_{t}+u m_{x}+2 m u_{x}=0, \quad \text { with } \quad u=g * m
$$

provides an invariant manifold on which canonical Hamiltonian dynamics occurs, for any choice of the Green's function $g$ relating velocity $u$ and momentum $m$ by the convolution $u=g * m$. 
The fluid velocity solutions corresponding to the singular momentum ansatz (37) for equation (51) are the pulsons. Pulsons are given by the sum over $N$ velocity profiles determined by the Green's function $g$, as

$$
u(x, t)=\sum_{a=1}^{N} p_{a}(t) g\left(x, q_{a}(t)\right) .
$$

Again for (51), the singular momentum ansatz (37) results in a finite-dimensional invariant manifold of solutions, whose dynamics is canonically Hamiltonian. The Hamiltonian for the canonical dynamics of the $2 N$ parameters $p_{a}(t)$ and $q_{a}(t)$ in the "pulson" solutions (52) of equation (51) is

$$
h_{N}=\frac{1}{2} \sum_{a, b=1}^{N} p_{a} p_{b} g\left(q_{a}, q_{b}\right) .
$$

Again for the pulsons, the canonical equations for the invariant manifold of singular momentum solutions provide a phase-space description of geodesic motion, this time with respect to the co-metric given by the Green's function $g$. Mathematical analysis and numerical results for the dynamics of these pulson solutions are given in [26]. These results describe how the collisions of pulsons (52) depend upon their shape.

\section{Compactons in the $1 / \alpha^{2} \rightarrow 0$ Limit of $\mathbf{C H}$}

As mentioned earlier, in the limit that $\alpha^{2} \rightarrow 0$, the $\mathrm{CH}$ equation (36) becomes the $\mathrm{KdV}$ equation. In the opposite limit that $1 / \alpha^{2} \rightarrow 0 \mathrm{CH}$ becomes the Hunter-Zheng equation [37]

$$
\left(u_{t}+u u_{x}\right)_{x x}=\frac{1}{2}\left(u_{x}^{2}\right)_{x}
$$

This equation has "compacton" solutions, whose collision dynamics was studied numerically and put into the present context in [26]. The corresponding Green's function satisfies $-\partial_{x}^{2} g(x)=2 \delta(x)$, so it has the triangular shape, $g(x)=1-|x|$ for $|x|<1$, and vanishes otherwise, for $|x| \geq 1$. That is, the Green's function in this case has compact support; hence, the name "compactons" for these pulson solutions, which as a limit of the integrable $\mathrm{CH}$ equations are true solitons, solvable by IST. 


\section{Pulson Solutions of the Dispersionless $b$-equation}

Holm and Staley [36] give the pulson solutions of the traveling wave problem and their elastic collision properties for the dispersionless $b$-equation

$$
m_{t}+u m_{x}+b m u_{x}=0, \quad \text { with } \quad u=g * m
$$

with any (symmetric) Green's function $g$ and for any value of the parameter $b$. Numerically, pulsons and peakons are both found to be stable for $b>1$, [36]. The reduction to noncanonical Hamiltonian dynamics for the invariant manifold of singular momentum solutions (37) of the other integrable case $b=3$ with peakon Green's function $g(x, y)=\mathrm{e}^{-|x-y| / \alpha}$ is found in [19].

\section{Euler-Poincaré Theory in Higher Dimensions}

\section{Generalizing the Peakon Solutions of the CH Equation to Higher Dimensions}

In [36], weakly nonlinear analysis and the assumption of columnar motion in the variational principle for Euler's equations were found to produce the twodimensional generalization of the dispersionless $\mathrm{CH}$ equation (38). This generalization is the Euler-Poincaré (EP) equation [35] for the Lagrangian consisting of the kinetic energy

$$
\ell=\frac{1}{2} \int\left[|\mathbf{u}|^{2}+\alpha^{2}(\operatorname{div} \mathbf{u})^{2}\right] \mathrm{d} x \mathrm{~d} y
$$

in which the fluid velocity $\mathbf{u}$ is a two-dimensional vector. Evolution generated by kinetic energy in Hamilton's principle results in geodesic motion, with respect to the velocity norm $\|\mathbf{u}\|$, which is provided by the kinetic energy Lagrangian. For ideal incompressible fluids governed by Euler's equations, the importance of geodesic flow was recognized by Arnold [4] for the $L^{2}$ norm of the fluid velocity. The EP equation generated by any choice of kinetic energy norm without imposing incompressibility is called "EPDiff," for "Euler-Poincaré equation for geodesic motion on the diffeomorphisms." EPDiff is given by [35]

$$
\left(\frac{\partial}{\partial t}+\mathbf{u} \cdot \nabla\right) \mathbf{m}+\nabla \mathbf{u}^{T} \cdot \mathbf{m}+\mathbf{m}(\operatorname{div} \mathbf{u})=0
$$

with momentum density $\mathbf{m}=\delta \ell / \delta \mathbf{u}$, where $\ell=\frac{1}{2}\|\mathbf{u}\|^{2}$ is given by the kinetic energy, which defines a norm in the fluid velocity $\|\mathbf{u}\|$, yet to be determined. By design, this equation has no contribution from either potential energy, or pressure. It conserves the velocity norm $\|\mathbf{u}\|$ given by the kinetic energy. Its evolution describes geodesic motion on the diffeomorphisms with respect to this norm [35]. An 
alternative way of writing the EPDiff equation (54) in either two, or three dimensions is

$$
\frac{\partial}{\partial t} \mathbf{m}-\mathbf{u} \times \operatorname{curl} \mathbf{m}+\nabla(\mathbf{u} \cdot \mathbf{m})+\mathbf{m}(\operatorname{div} \mathbf{u})=0 .
$$

This form of EPDiff involves all three differential operators, curl, gradient and divergence. For the kinetic energy Lagrangian $\ell$ given in (53), which is a norm for irrotational flow (with curl $\mathbf{u}=0$ ), we have the EPDiff equation (54) with momentum $\mathbf{m}=\delta \ell / \delta \mathbf{u}=\mathbf{u}-\alpha^{2} \nabla(\operatorname{div} \mathbf{u})$.

EPDiff (54) may also be written intrinsically as

$$
\frac{\partial}{\partial t} \frac{\delta \ell}{\delta \mathbf{u}}=-\operatorname{ad}_{\mathbf{u}}^{*} \frac{\delta \ell}{\delta \mathbf{u}}
$$

where $\mathrm{ad}^{*}$ is the $L^{2}$ dual of the ad-operation (commutator) for vector fields. See [5, 44] for additional discussions of the beautiful geometry underlying this equation.

\section{Reduction to the Dispersionless CH Equation in 1D}

In one dimension, the EPDiff equation (54-56) with Lagrangian $\ell$ given in (53) simplifies to the dispersionless $\mathrm{CH}$ equation (38). The dispersionless limit of the $\mathrm{CH}$ equation appears, because we have ignored potential energy and pressure.

\section{Strengthening the Kinetic Energy Norm to Allow for Circulation}

The kinetic energy Lagrangian (53) is a norm for irrotational flow, with curl $\mathbf{u}=0$. However, inclusion of rotational flow requires the kinetic energy norm to be strengthened to the $H_{\alpha}^{1}$ norm of the velocity, defined as

$$
\begin{aligned}
\ell & =\frac{1}{2} \int\left[|\mathbf{u}|^{2}+\alpha^{2}(\operatorname{div} \mathbf{u})^{2}+\alpha^{2}(\operatorname{curl} \mathbf{u})^{2}\right] \mathrm{d} x \mathrm{~d} y \\
& =\frac{1}{2} \int\left[|\mathbf{u}|^{2}+\alpha^{2}|\nabla \mathbf{u}|^{2}\right] \mathrm{d} x \mathrm{~d} y=\frac{1}{2}\|\mathbf{u}\|_{H_{\alpha}^{1}}^{2} .
\end{aligned}
$$

Here we assume some boundary conditions that give no contributions upon integrating by parts. The corresponding EPDiff equation is (54) with $\mathbf{m} \equiv \delta \ell / \delta \mathbf{u}=$ $\mathbf{u}-\alpha^{2} \Delta \mathbf{u}$. This expression involves inversion of the familiar Helmholtz operator in the (nonlocal) relation between fluid velocity and momentum density. The $H_{\alpha}^{1}$ norm $\|\mathbf{u}\|_{H_{\alpha}^{1}}^{2}$ for the kinetic energy (58) also arises in three dimensions for turbulence modeling based on Lagrangian averaging and using Taylor's hypothesis that the turbulent fluctuations are "frozen" into the Lagrangian mean flow [22]. 


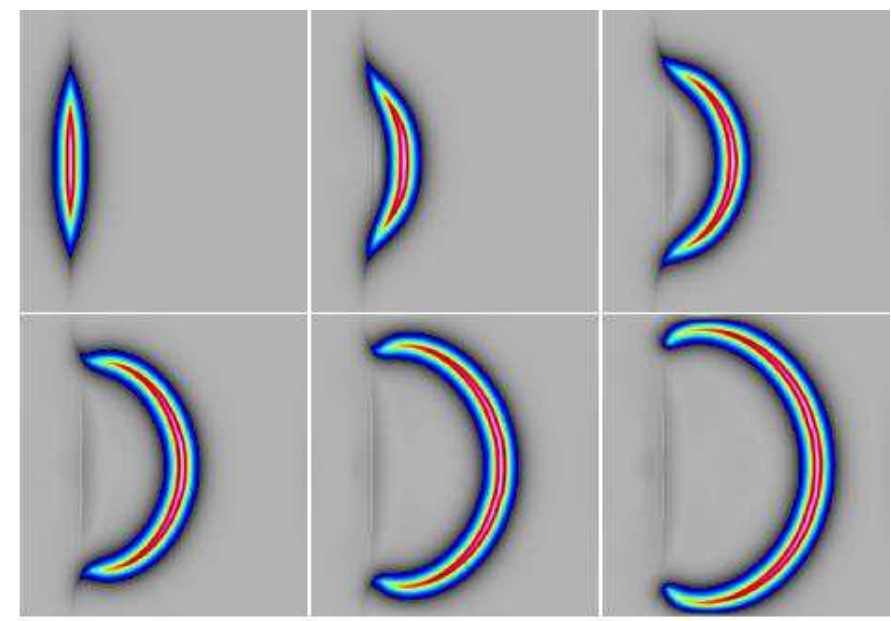

Figure 3. A peakon segment of finite length is initially moving rightward (East). Because its speed vanishes at its ends and it has fully two-dimensional spatial dependence, it expands into a peakon "bubble" as it propagates. (The colors indicate speed: red is highest, yellow is less, blue low, grey zero.)

\section{Generalizing the CH Peakon Solutions to $n$ Dimensions.}

Building on the peakon solutions (35) for the $\mathrm{CH}$ equation and the pulsons (52) for its generalization to other traveling-wave shapes in [26], Holm and Staley [36] introduced the following measure-valued singular momentum solution ansatz ${ }^{2}$ for the $n$-dimensional solutions of the EPDiff equation (54)

$$
\mathbf{m}(\mathbf{x}, t)=\sum_{a=1}^{N} \int \mathbf{P}^{a}(s, t) \delta\left(\mathbf{x}-\mathbf{Q}^{a}(s, t)\right) \mathrm{d} s .
$$

These singular momentum solutions, called "diffeons," are vector density functions supported in $\mathbb{R}^{n}$ on a set of $N$ surfaces (or curves) of codimension $(n-k)$ for $s \in \mathbb{R}^{k}$ with $k<n$. They may, for example, be supported on sets of points (vector peakons, $k=0$ ), one-dimensional filaments (strings, $k=1$ ), or two-dimensional surfaces (sheets, $k=2$ ) in three dimensions.

Figure 3 shows the results for the EPDiff equation when a straight peakon segment of finite length is created initially moving rightward (East). Because of propagation along the segment in adjusting to the condition of zero speed at its ends and finite speed in its interior, the initially straight segment expands outward as it propagates and curves into a peakon "bubble."

\footnotetext{
${ }^{2}$ These solutions represent smooth embeddings $\operatorname{Emb}\left(\mathbb{R}^{k}, \mathbb{R}^{n}\right)$ with $k<n$. In contrast, the similar expression (30) for the soliton solutions represent smooth functions $\mathbb{R} \rightarrow \mathbb{R}$.
} 


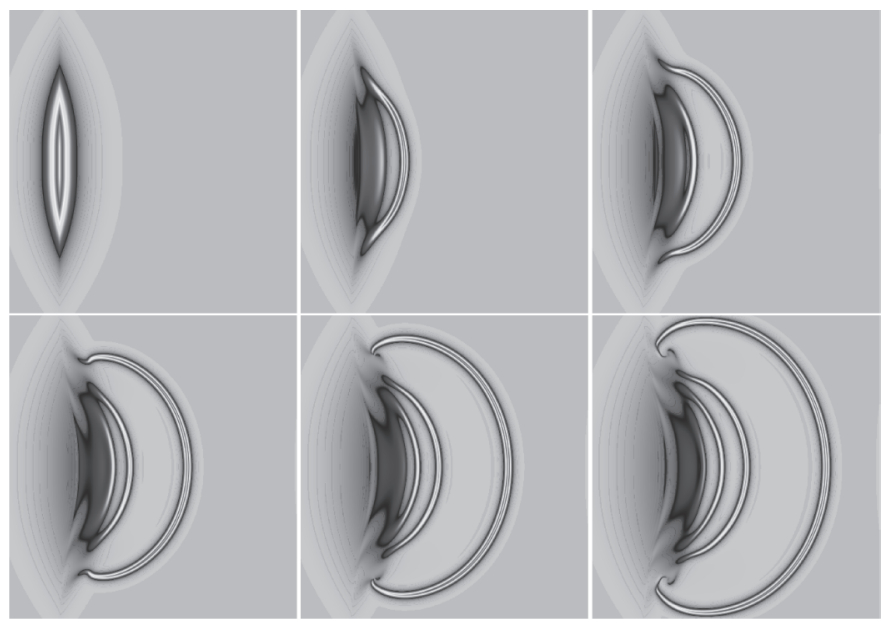

Figure 4. An initially straight segment of velocity distribution whose exponential profile is wider than the width $\alpha$ for the peakon solution will break up into a train of curved peakon "bubbles," each of width $\alpha$. This example illustrates the emergent property of the peakon solutions in two dimensions.

Figure 4 shows an initially straight segment whose velocity distribution is exponential in the transverse direction, but is wider than $\alpha$ for the peakon solution. This initial velocity distribution evolves under EPDiff to separate into a train of curved peakon "bubbles," each of width $\alpha$. This example illustrates the emergent property of the peakon solutions in two dimensions. This phenomenon is observed in nature, for example, as trains of internal wave fronts in the south China Sea [43].

Substitution of the singular momentum solution ansatz (58) into the EPDiff equation (54) implies the following integro-partial-differential equations (IPDEs) for the evolution of the parameters $\{\mathbf{P}\}$ and $\{\mathbf{Q}\}$

$$
\begin{aligned}
& \frac{\partial}{\partial t} \mathbf{Q}^{a}(s, t)=\sum_{b=1}^{N} \int \mathbf{P}^{b}\left(s^{\prime}, t\right) G\left(\mathbf{Q}^{a}(s, t)-\mathbf{Q}^{b}\left(s^{\prime}, t\right)\right) \mathrm{d} s^{\prime} \\
& \frac{\partial}{\partial t} \mathbf{P}^{a}(s, t)=-\sum_{b=1}^{N} \int\left(\mathbf{P}^{a}(s, t) \cdot \mathbf{P}^{b}\left(s^{\prime}, t\right)\right) \\
& \frac{\partial}{\partial \mathbf{Q}^{a}(s, t)} G\left(\mathbf{Q}^{a}(s, t)-\mathbf{Q}^{b}\left(s^{\prime}, t\right)\right) \mathrm{d} s^{\prime} .
\end{aligned}
$$




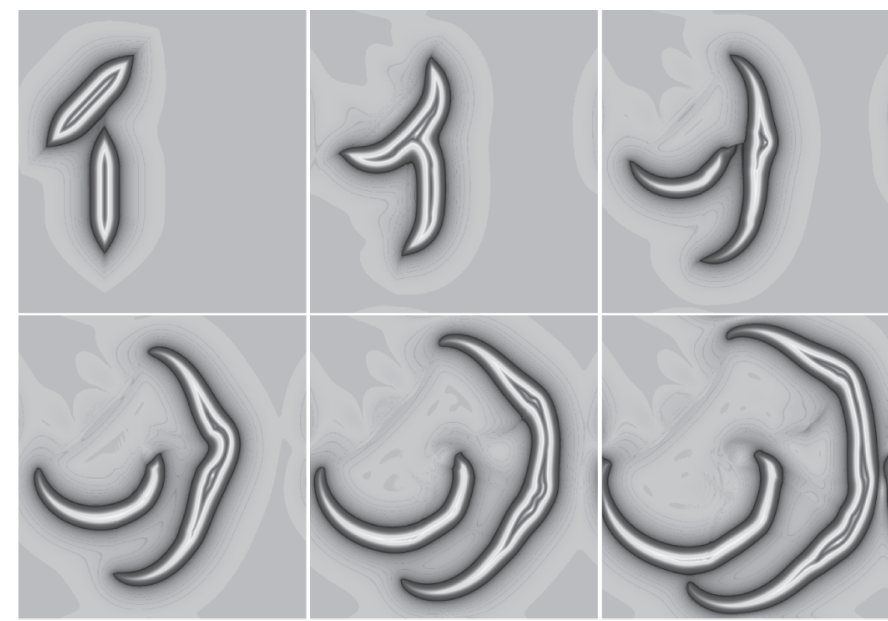

Figure 5. A single collision is shown involving reconnection as the faster peakon segment initially moving Southeast along the diagonal expands, curves and obliquely overtakes the slower peakon segment initially moving rightward (East). This reconnection illustrates one of the collision rules for the strongly two-dimensional EPDiff flow.

Importantly for the interpretation of these solutions, the coordinates $s \in \mathbb{R}^{k}$ turn out to be Lagrangian coordinates. The velocity field corresponding to the momentum solution ansatz (58) is given by

$$
\mathbf{u}(\mathbf{x}, t)=G * \mathbf{m}=\sum_{b=1}^{N} \int \mathbf{P}^{b}\left(s^{\prime}, t\right) G\left(\mathbf{x}-\mathbf{Q}^{b}\left(s^{\prime}, t\right)\right) \mathrm{d} s^{\prime}
$$

for $\mathbf{u} \in \mathbb{R}^{n}$. When evaluated along the curve $\mathbf{x}=\mathbf{Q}^{a}(s, t)$, this velocity satisfies,

$$
\begin{aligned}
\mathbf{u}\left(\mathbf{Q}^{a}(s, t), t\right) & =\sum_{b=1}^{N} \int \mathbf{P}^{b}\left(s^{\prime}, t\right) G\left(\mathbf{Q}^{a}(s, t)-\mathbf{Q}^{b}\left(s^{\prime}, t\right)\right) \mathrm{d} s^{\prime} \\
& =\frac{\partial \mathbf{Q}^{a}(s, t)}{\partial t} .
\end{aligned}
$$

Consequently, the lower-dimensional support sets defined on $\mathbf{x}=\mathbf{Q}^{a}(s, t)$ and parameterized by coordinates $s \in \mathbb{R}^{k}$ move with the fluid velocity. This means the $s \in \mathbb{R}^{k}$ are Lagrangian coordinates. Moreover, equations (59) for the evolution of these support sets are canonical Hamiltonian equations

$$
\frac{\partial}{\partial t} \mathbf{Q}^{a}(s, t)=\frac{\delta H_{N}}{\delta \mathbf{P}^{a}}, \quad \frac{\partial}{\partial t} \mathbf{P}^{a}(s, t)=-\frac{\delta H_{N}}{\delta \mathbf{Q}^{a}} .
$$




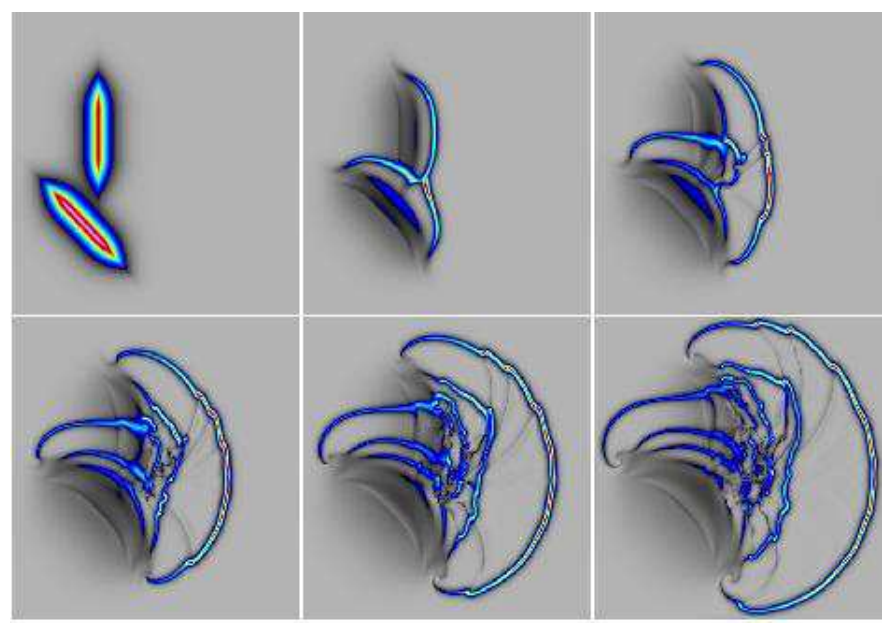

Figure 6. A series of multiple collisions is shown involving reconnections as the faster wider peakon segment initially moving Northeast along the diagonal expands, breaks up into a wave train of peakons, each of which propagates, curves and obliquely overtakes the slower wide peakon segment initially moving rightward (East), which is also breaking up into a train of wavefronts. In this series of oblique collision, the now-curved peakon filaments exchange momentum and reconnect several times.

The corresponding Hamiltonian function $H_{N}:\left(\mathbb{R}^{n} \times \mathbb{R}^{n}\right)^{N} \rightarrow \mathbb{R}$ is

$$
H_{N}=\frac{1}{2} \iint \sum_{a, b=1}^{N} \mathbf{P}^{a}(s, t) \cdot \mathbf{P}^{b}\left(s^{\prime}, t\right) G\left(\mathbf{Q}^{a}(s, t), \mathbf{Q}^{b}\left(s^{\prime}, t\right)\right) \mathrm{d} s \mathrm{~d} s^{\prime}
$$

This is the Hamiltonian for geodesic motion on the cotangent bundle of a set of curves $\mathbf{Q}^{a}(s, t)$ with respect to the metric given by $G$. This dynamics was investigated numerically in [36] to which we refer for more details of the solution properties. One important result found numerically in [36] is that only codimension-one singular momentum solutions appear to be stable under the evolution of the EPDiff equation. Thus, we have

Stability for codimension-one: the singular momentum solutions of EPDiff are stable, as points on the line (peakons), as curves in the plane (filaments, or wave fronts), or as surfaces in space (sheets).

Proving this stability result analytically remains an outstanding problem. The stability of peakons on the real line is proven in [17]. 


\section{Reconnections in Oblique Overtaking Collisions of Peakon Wave Fronts}

Figure 5 and Figure 6 shows results of oblique wave front collisions producing reconnections for the EPDiff equation in two dimensions. Figure 5 show a single oblique overtaking collision, as a faster expanding peakon wave front overtakes a slower one and reconnects with it at the collision point.

\section{The Peakon Solution Ansatz is a Momentum Map}

As shown in [34], the solution expressions (37) in one dimension and (58) in higher dimensions may be interpreted as equivariant momentum maps, from the cotangent bundle of the smooth embeddings of lower dimensional sets $\mathbb{R}^{s} \subset \mathbb{R}^{n}$, to the dual of the Lie algebra of vector fields defined on these sets. (Momentum maps for Hamiltonian dynamics are reviewed in [44], for example.) The result that the singular solution ansatz (58) is a momentum map helps to organize the theory, to explain previous results and to suggest new avenues of exploration. This geometric feature underlies the remarkable reduction properties of the $\mathrm{CH}$ and EPDiff equations, and explains why they must be Lie-Poisson Hamiltonian equations. This is because of the general fact that equivariant momentum maps are Poisson maps. This geometric feature also underlies the singular momentum solution (58) and its associated velocity (60) which generalize the peakon solutions, both to higher dimensions and to arbitrary kinetic energy metrics. As we saw in Section 3, the soliton solution (33) is also a momentum map. This soliton momentum map may be expected to apply in the action-angle representation of the solution of any integrable Hamiltonian PDE. Its further properties will be studied in detail elsewhere.

\section{Two Open Problems}

1. Throughout this discussion the solutions $u(x, t)$ were confined to be functions in the Schwartz class, $\omega>0$. The situation when the condition $m(x, 0)+\omega>0$ on the initial data does not hold is more complicated and requires separate analysis $[10,12,40]$. In general, it leads to wave-breaking [12]. An attempt at developing the inverse scattering theory for this case has been made by Kaup [40], who suggested applying the inverse scattering approach separately in each interval where $m(x, t)+\omega$ is of the same sign. The problem however is how to join solutions that are valid in different intervals.

2. Stability for EPDiff singular momentum solutions, that is, proving their stability analytically, remains an outstanding problem. 


\section{Acknowledgements}

The first named author is quite grateful to R. Camassa, J. E. Marsden, T. S. Ratiu and A. Weinstein for their collaboration, help and inspiring discussions over the years and gratefully acknowledges partial support by the Royal Society of London's Wolfson Award scheme. The second named author acknowledges funding from a Marie Curie Intra-European Fellowship. Both authors thank J. Percival and M. F. Staley for providing figures.

\section{References}

[1] Ablowitz M. and Clarkson P., Solitons, Nonlinear Evolution Equations and Inverse Scattering, Cambridge Univ. Press, Cambridge, 1991.

Ablowitz M. and Segur H., Solitons and the Inverse Scattering Transform, SIAM, Philadelphia, 1981.

[2] Agrotis M., Damianou P. and Christodulos S., The Toda Lattice is Superintegrable, Physica A 365 (2006) 235-243.

[3] Alber M., Camassa R., Fedorov Y., Holm D. and Marsden J., On Billiard Solutions of Nonlinear PDE's, Phys. Lett. A 264 (1999) 171-178.

Alber M., Camassa R., Fedorov Y., Holm D. and Marsden J., The Complex Geometry of Weak Piecewise Smooth Solutions of Integrable Nonlinear PDE's of Shallow Water and Dym Type, Comm. Math. Phys. 221 (2001) 197227.

Alber M., Camassa R., Holm D. and Marsden J., The Geometry of Peaked Solitons and Billiard Solutions of a Class of Integrable PDE's, Lett. Math. Phys. 32 (1994) 137-151.

Alber M., Camassa R. and Gekhtman M., On Billiard Weak Solutions of Nonlinear PDE's and Toda Flows, CRM Lecture Notes 25 (2000) 1-11.

[4] Arnold V., Sur la geometrie differentielle des groupes de Lie de dimension infinie et ses applications a l'hydrodynamique des fluides parfaits, Ann. Inst. Fourier 16 (1966) 319-361.

[5] Arnold V. and Khesin B., Topological Methods in Hydrodynamics, Springer, New York, 1998.

[6] Beals R., Sattinger D. and Szmigielski J., Multi-peakons and a Theorem of Stieltjes, Inverse Problems 15 (1999) L1-L4.

Beals R., Sattinger D. and Szmigielski J., Multipeakons and the Classical Moment Problem, Adv. Math. 154 (2000) 229-257. 
Beals R., Sattinger D. and Szmigielski J., Peakons, Strings, and the Finite Toda Lattice, Commun. Pure Appl. Math. 54 (2001) 91-106.

[7] Birkhoff G. and Rota G.-C., Ordinary Differential Equations, Blaisdell Publishing Company, Waltham 1969.

[8] Calogero F., An Integrable Hamiltonian System, Phys. Lett. A 201 (1995) 306-310.

Calogero F. and Francoise J-P., A Completely Integrable Hamiltonian System, J. Math. Phys. 37 (1996) 2863-2871.

[9] Camassa R. and Holm D., An Integrable Shallow Water Equation with Peaked Solitons, Phys. Rev. Lett. 71 1661-1664; ArXiv: patt-sol/9305002.

Camassa R., Holm D. and Hyman J., A New Integrable Shallow Water Equation, Adv. Appl. Mech. 31 (1994) 1-33.

[10] Constantin A., On the Scattering Problem for the Camassa-Holm Equation, Proc. R. Soc. Lond. A (2001) 457 953-970.

[11] Constantin A., Finite Propagation Speed for the Camassa-Holm Equation, J. Math. Phys. 46 (2005) 023506 (4 pages).

[12] Constantin A. and Escher J., Wave Breaking for Nonlinear Nonlocal Shallow Water Equations, Acta Mathematica 181 (1998) 229-243.

[13] Constantin A., Gerdjikov V. and Ivanov R., Inverse Scattering Transform for the Camassa-Holm Equation, Inv. Problems 22 (2006) 2197-2207, arXiv:nlin/0603019v2 [nlin.SI].

[14] Constantin A., Gerdjikov V. and Ivanov R., Generalised Fourier Transform for the Camassa-Holm Hierarchy, Inv. Problems 23 (2007) 1565-1597, arXiv:nlin.SI/0707.2048.

[15] Constantin A. and Ivanov R., Poisson Structure and Action-Angle Variables for the Camassa-Holm Equation, Lett. Math. Phys. 76 (2006) 93-108, arXiv:nlin.SI/0602049.

[16] Constantin A. and McKean H. A Shallow Water Equation on the Circle, Commun. Pure Appl. Math. 52 (1999) 949-982.

[17] Constantin A. and Strauss W., Stability of Peakons, Commun. Pure Appl. Math. 53 (2000) 603-610.

[18] Dai H.-H., Model Equations for Nonlinear Dispersive Waves in a Compressible Mooney-Rivlin Rod, Acta Mech. 127 (1998) 193-207.

[19] Degasperis A. and Procesi M., Asymptotic Integrability, In: Symmetry and Perturbation Theory, A. Degasperis and G. Gaeta (Eds), World Scientific, Singapore 1999, pp 23-37. 
Degasperis A., Holm D. and Hone A., A New Integrable Equation with Peakon Solutions, Theor. Math. Phys. 133 (2002) 1463-1474.

[20] Dubrovin B., Theta Functions and Non-linear Equations, Russ. Math. Surv. 36 (1981) 11-92.

Dubrovin B., Novikov S. and Krichever I., Integrable Systems. I (in Russian), In: Itogi Nauki i Tekhniki. Sovr. Probl. Mat. Fund. Naprav. 4 VINITI, Moscow, 1985.

Dubrovin B., Novikov S. and Krichever I., Encyclopaedia of Mathematical Sciences 4 (Engl. Transl.), Springer, Berlin 1989.

[21] Dullin H., Gottwald G. and Holm D., An Integrable Shallow Water Equation with Linear and Nonlinear Dispersion, Phys. Rev. Lett. 87 (2001) 194501 (4 pages), arXiv: nlin.CD/0104004;

Dullin H., Gottwald G. and Holm D., Camassa-Holm, Korteweg-de Vries-5 and Other Asymptotically Equivalent Eequations for Shallow Water Waves, Fluid Dyn. Res. 33 (2003) 73-95.

Dullin H., Gottwald G. and Holm D., On Asymptotically Equivalent Shallow Water Wave Equations, Physica D 190 (2004) 1-14.

[22] Foias C., Holm D. and Titi E., The Navier-Stokes-alpha Model of Fluid Turbulence, Physica D 152 (2001) 505-519.

[23] Fokas A. and Fuchssteiner B., Bäcklund Transformations for Hereditary Symmetries, Nonlinear Anal. TMA 5 (1981) 423-432.

[24] Fokas A. and Liu Q., Asymptotic Integrability of Water Waves, Phys. Rev. Lett. 77 (1996) 2347-2351.

[25] Fuchssteiner B., Some Tricks from the Symmetry-Toolbox for Nonlinear Equations, Physica D 95 (1996) 229-243.

[26] Fringer O. and Holm D., Integrable vs. Nonintegrable Geodesic Soliton Behavior, Physica D 150 (2001) 237-263, arXiv:solv-int/9903007.

[27] Gardner C., Korteweg-de Vries Equation and Generalizations. IV. The Korteweg-de Vries Equation as a Hamiltonian System, J. Mathematical Phys. 12 (1971) 1548-1552.

[28] Gardner C., Greene J., Kruskal M. and Miura R., Method for Solving the Korteweg-deVries Equation, Phys. Rev. Lett. 19 (1967) 1095-1097.

[29] Gardner C., Greene J., Kruskal M. and Miura R., Korteweg-de Vries Equation and Generalizations. VI. Methods for Exact Solution, Comm. Pure Appl. Math. 27 (1974) 97-133. 
[30] Gerdjikov, V., On Spectral Theory of Lax Operators on Symmetric Spaces: Vanishing Versus Constant Boundary Conditions, J. Geom. Symmetry Phys. 15 (2009) 1-41.

[31] Gerdjikov V., Evstatiev E. and Ivanov R., The Complex Toda Chains and the Simple Lie Algebras - Solutions and Large Time Asymptotics, J. Phys. A: Math \& Gen. 31 (1998) 8221-8232, ArXiv:solv-int/9712004.

[32] Gerdjikov V., Evstatiev E. and Ivanov R., The Complex Toda Chains and the Simple Lie Algebras II. Explicit Solutions and Asymptotic Behaviour, J. Phys. A: Math \& Gen. 33 (2000) 975-1006.

[33] Gerdjikov V., Kaup D., Uzunov I. and Evstatiev E., Asymptotic Behavior of N-Soliton Trains of the Nonlinear Schrödinger Equation, Phys. Rev. Lett. 77 (1996) 3943-3946.

[34] Holm D. and Marsden J., Momentum Maps and Measure-valued Solutions (Peakons, Filaments and Sheets) for the EPDiff Equation, In: The Breadth of Symplectic and Poisson Geometry, Progr. Math. Series vol. 232, J. Marsden and T. Ratiu (Eds), Birkhäuser, Boston 2004, pp 203-235.

[35] Holm D., Marsden J. and Ratiu T., The Euler-Poincaré Equations and Semidirect Products with Applications to Continuum Theories, Adv. Math. 137 (1998) 1-81, arXiv: chao-dyn/9801015.

Holm D., Marsden J. and Ratiu T., Euler-Poincaré Models of Ideal Fluids with Nonlinear Dispersion, Phys. Rev. Lett. 349 (1998) 4173-4176.

[36] Holm D. and Staley M., Nonlinear Balance and Exchange of Stability in Dynamics of Solitons, Peakons, Ramps/Cliffs and Leftons in a $1+1$ Nonlinear Evolutionary PDE, Phys. Lett. A 308 (2003) 437-444.

Holm D. and Staley M., Wave Structure and Nonlinear Balances in a Family of Evolutionary PDEs, SIAM J. Appl. Dyn. Syst. 2 (2003) 323-380.

[37] Hunter J. and Zheng Y. On a Completely Integrable Nonlinear Hyperbolic Variational Equation, Physica D 79 (1994) 361-386.

[38] Johnson R., Camassa-Holm, Korteweg-de Vries and Related Models for Water Waves, J. Fluid Mech. 455 (2002) 63-82.

[39] Johnson R., The Camassa-Holm Equation for Water Waves Moving over a Shear Flow, Fluid Dynamics Research 33 (2003) 97-111.

[40] Kaup D., Evolution of the Scattering Coefficients of the Camassa-Holm Equation, for General Initial Data, Stud. Appl. Math. 117 (2006) 149-164.

[41] Kodama Y., On Integrable Systems With Higher Order Corrections, Phys. Lett. A 107 (1985) 245-249. 
Kodama Y., Normal Forms for Weakly Dispersive Wave Equations, Phys. Lett. A 112 (1985) 193-196.

Kodama Y., On Solitary-wave Interaction, Phys. Lett. A 123 (1987) 276-282.

[42] Lax P., Integrals of Nonlinear Equations of Evolution and Solitary Waves, Comm. Pure Appl. Math. 21 (1968) 467-490.

[43] Liu A., Chang Y., Hsu M.-K. and Liang N., Evolution of Nonlinear Internal Waves in the East and South China Seas, J. Geophys. Res. 103 (1998) 79958008 .

[44] Marsden J. and Ratiu T., Introduction to Mechanics and Symmetry, Texts in Applied Mathematics 17, Second Edition, Springer, Berlin 1999.

[45] Matsuno Y., Parametric Representation for the Multisoliton Solution of the Camassa-Holm Equation, J. Phys. Soc. Japan 74 (2005) 1983-1987, ArXiv:nlin.SI/0504055.

[46] Matsuno Y., The Peakon Limit of the N-Soliton Solution of the CamassaHolm Equation, J. Phys. Soc. Japan 76 (2007) 034003 (8 pages), ArXiv:nlin/0701051.

[47] Mikhailov A. and Novikov V., Perturbative Symmetry Approach, J. Phys. A:Math \& Gen. 35 (2002) 4775-4790.

[48] Novikov S., Manakov S., Pitaevskii L. and Zakharov V., Theory of Solitons: the Inverse Scattering Method (translated from Russian), Plenum, New York, 1984.

[49] Parker A., On the Camassa-Holm Equation and a Direct Method of Solution I. Bilinear Form and Solitary Waves, Proc. R. Soc. Lond. A 460 (2004) 29292957.

[50] Parker A., On the Camassa-Holm Equation and a Direct Method of Solution. II. Soliton Solutions, Proc. R. Soc. Lond. A 461 (2005) 3611-3632.

[51] Parker A., On the Camassa-Holm Equation and a Direct Method of Solution. III. N-soliton Solutions, Proc. R. Soc. Lond. A 461 (2005) 3893-3911.

[52] Parker A., Wave Dynamics for Peaked Solitons of the Camassa-Holm Equation, Chaos, Solitons and Fractals 35 (2008) 220-237.

[53] Ragnisco O. and Bruschi M., Peakons, r-matrix and Toda Lattice, Physica A 228 (1996) 150-159.

[54] Suris Y., A Discrete Time Peakons Lattice, Phys. Lett. A 217 (1996) 321-329.

[55] Toda M., Vibration of a Chain with Nonlinear Interaction, J. Phys Soc. Japan 22 (1967) 431-436. 
[56] Zakharov V. and Faddeev L., Korteweg-de Vries Equation: A Completely Integrable Hamiltonian System (English translation), Func. Anal. Appl. 5 (1971) 280-287; Funkz. Anal. Prilož. 5 (1971) 18-25 (in Russian).

Darryl D. Holm

Department of Mathematics

Imperial College London

London SW7 2AZ, UK

E-mail address: d.holmeimperial.ac.uk

Rossen I. Ivanov

Department of Mathematics

Imperial College London

London SW7 2AZ, UK

and

School of Mathematical Sciences

Dublin Institute of Technology

Kevin Street, Dublin 8, IRELAND

E-mail address: r.ivanov@imperial.ac.uk

rivanovedit.ie 